\title{
The role of magnetic resonance imaging in the evaluation of bone tumours and tumour-like lesions
}

\author{
Duarte Nascimento • Guilherme Suchard • \\ Maruan Hatem • Armando de Abreu
}

Received: 20 April 2014 /Revised: 11 May 2014 / Accepted: 20 May 2014 /Published online: 9 July 2014

(C) The Author(s) 2014. This article is published with open access at Springerlink.com

\begin{abstract}
Bone tumours and tumour-like lesions are frequently encountered by radiologists. Although radiographs are the primary screening technique, magnetic resonance imaging (MRI) can help narrow the differential or make a specific diagnosis when a lesion is indeterminate or shows signs of aggressiveness. MRI can extend the diagnostic evaluation by demonstrating several tissue components. Even when a specific diagnosis cannot be made, the differential diagnosis can be narrowed. MRI is superior to the other imaging modalities in detecting bone marrow lesions and tumoral tissue (faint lytic/sclerotic bone lesions can be difficult to visualise using only radiographs). Contrast-enhanced MRI can reveal the most vascularised parts of the tumour and MRI guidance makes it possible to avoid biopsing necrotic areas. MRI is very helpful in local staging and surgical planning by assessing the degree of intramedullary extension and invasion of the adjacent physeal plates, joints, muscle compartments and neurovascular bundles. It can be used in assessing response to neoadjuvant therapy and further restaging. The posttherapeutic follow-up should also be done using MRI. Despite
\end{abstract}

D. Nascimento $(\bowtie)$

Serviço de Imagiologia do Serviço de Saúde da Região Autónoma da Madeira, Avenida Luís de Camões, nº 57, 9004-514 Funchal,

Portugal

e-mail: duarterufino@gmail.com

G. Suchard $\cdot$ M. Hatem $\cdot$ A. de Abreu

Serviço de Radiologia do Hospital Mãe de Deus, Rua José de

Alencar, 286, Menino Deus, Porto Alegre RS, 90880-480, Brazil

G. Suchard

e-mail: guisuchard@gmail.com

M. Hatem

e-mail: maruanhatem@hotmail.com

A. de Abreu

e-mail: armandoabreu@terra.com.br the high quality of MRI, there are a few pitfalls and limitations of which one should be aware. Applications of MRI in bone tumours will probably continue to grow as new sequences are further studied.

\section{Teaching Points}

- When a lesion is indeterminate or shows signs of aggressiveness, MRI is indicated.

- When MRI does not lead to a diagnosis, biopsy is indicated.

- MRI is superior to the other imaging modalities in detecting bone marrow lesions.

- MRI is very helpful in local staging and surgical planning.

- MRI is used in assessing the response to neoadjuvant therapy, restaging and post-therapeutic follow-up.

Keywords Magnetic resonance imaging - Bone neoplasms · Diagnosis $\cdot$ Neoplasm staging $\cdot$ Follow-up

\section{Introduction}

Radiographs are the primary screening technique used for bone tumours and tumour-like lesions [1]. When a lesion is indeterminate or shows signs of aggressiveness, magnetic resonance imaging (MRI) is indicated for further characterisation [1]. It can extend the diagnostic evaluation by demonstrating components such as cartilage, vascular tissue, fat, liquid and haemosiderin. Even when a specific diagnosis cannot be made, MRI can help by narrowing the differential diagnosis. These are the reasons why MRI has changed from a single study-based diagnosis (solely based on radiographs) to a multimodal imaging approach (which now includes MRI).

Faint lytic/sclerotic bone lesions can be difficult to visualise using only radiographs. MRI is superior to the other imaging modalities in detecting bone marrow lesions [2]. 
Fig. 1 This femoral lesion showing high signal in T2FS WI (a) could be solid (fibrous dysplasia) or liquid (solitary bone cyst). T1FS gadolinium-based contrast medium-enhanced sequence (b) showed peripheral enhancement, typical of liquid content, favouring the diagnosis of a solitary bone cyst
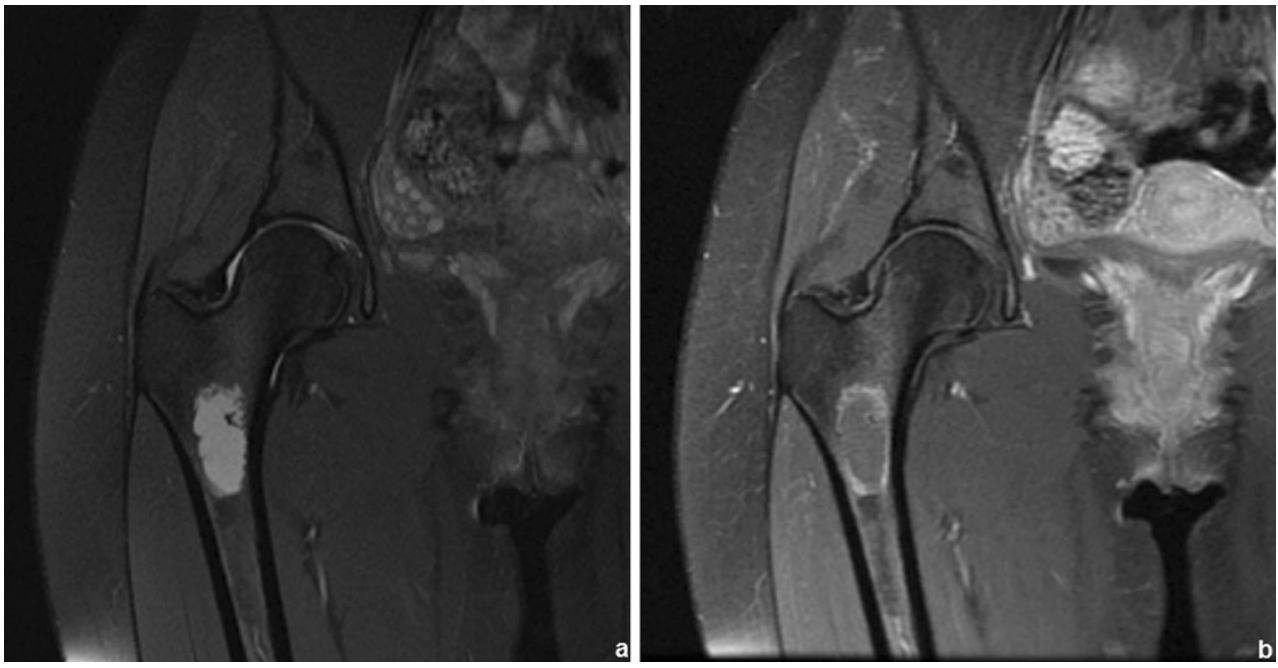

Aggressive indeterminate cases will require histological confirmation before proceeding to staging and establishing a therapeutic approach. The high percentage of biopsy tract contamination [3] indicates that this track should be included in the surgically removed area. Contrast-enhanced MRI (CEMRI) can reveal the most vascularised parts of the tumour and MRI guidance makes it possible to avoid biopsing necrotic areas [2].

MRI is very helpful in local staging and surgical planning because it assesses the degree of intramedullary extension (and dimensions) and invasion of the adjacent physeal plates, joints, muscle compartments and neurovascular bundles.

Restaging after neoadjuvant therapy and the posttherapeutic follow-up should also be done using MRI.

Our purposes are: (1) to discuss MRI features that can help narrow the differential or make a specific diagnosis of bone tumours and tumour-like lesions; (2) explain why MRI is the optimal imaging method for sensitive detection of tumoral tissue, local staging, preoperative evaluation, assessing the response to neoadjuvant therapy, restaging and follow-up, and (3) to discuss potential pitfalls and limitations.

\section{MR imaging sequences and protocol optimisation}

In musculoskeletal (MSK) MRI, the T1 signal intensity is described by comparison to that of muscle. Although many argue that muscle should also be the standard reference for $\mathrm{T} 2$-weighted imaging, using fat as the reference can be helpful, particularly in anatomic regions where there is relatively little muscle (e.g. fingers and toes).
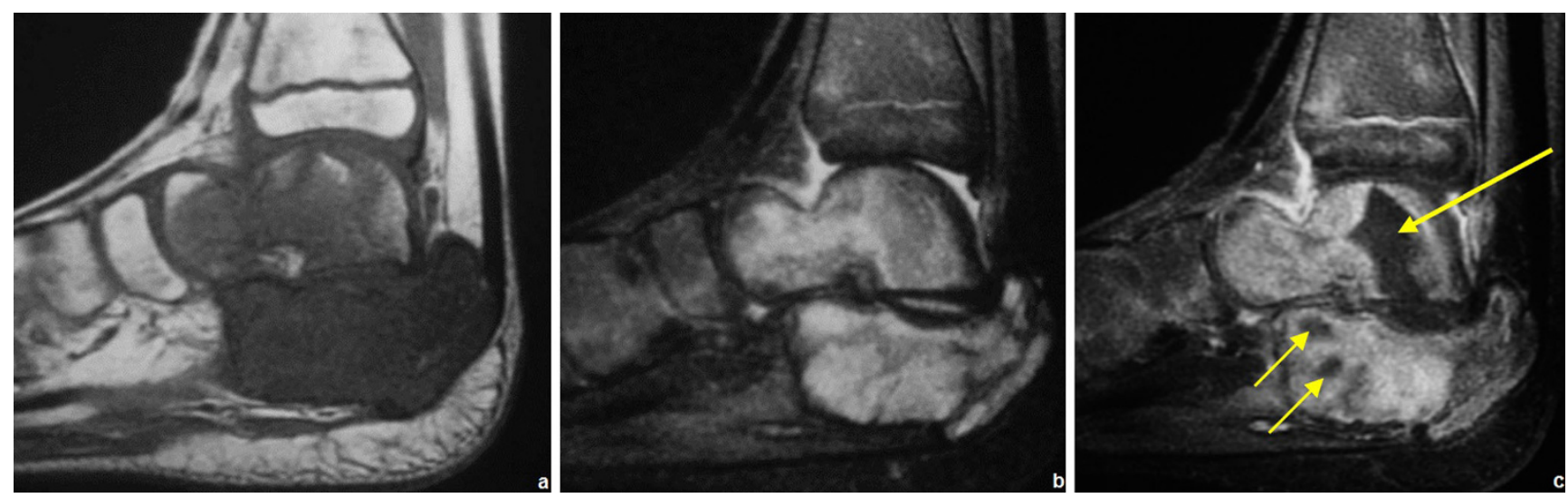

Fig. 2 T1WI (a), T2FS WI (b) and CEMRI (c) images of a Ewing's sarcoma involving the talus and calcaneus. Non-enhancing components (arrows in c) are related to areas of necrosis 

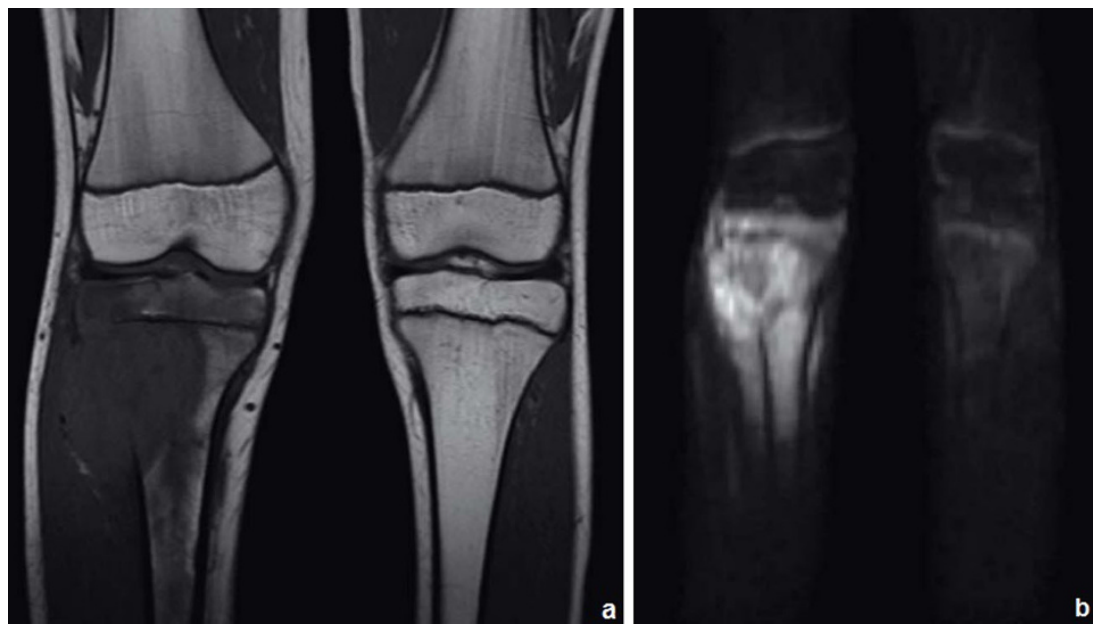

Fig. 3 Osteosarcoma of the tibia: coronal T1WI (a) shows a large hypointense lesion in the proximal tibia, which breaks through the bony cortex and invades the adjacent soft tissue. Corresponding DWI $(b=$ $700 \mathrm{~s} / \mathrm{mm}^{2}$ ) (b) reveals restricted proton diffusion, consistent with

\section{T1-weighted imaging (T1WI)}

T1WI is very important in the evaluation of bone marrow. Most bone tumours will be evident as lesions with low signal against a background of surrounding fatty marrow [4].

T1WI also provides excellent contrast among the cortical, marrow and surrounding tissues [4].

Fat suppression, T2WI and STIR

The use of fat suppression (FS) in MRI can confirm or exclude the presence of fat in a lesion (this is malignancy. Tumoral necrosis (in the centre of the tumour) displays a less hyperintense signal. (Images published by Markus U., Reichardt W. and Kontny U. in MRI: New Developments in Bone Tumor Imaging. Magnetom Flash 2/2011@ used with permission of Prof. U. Markus)

particularly useful for diagnosing haemangioma and lipoma).

Water shows higher signal than fat on T2WI, but suppressing the fat signal can allow an even better evaluation of the extent of oedema.

Suppressing the fat signal in T1WI after injection of gadolinium-based contrast medium increases the conspicuousness when assessing tumour vascularisation.

Short tau inversion recovery (STIR) sequences effectively and homogeneously suppress all fat signal but cause tissues with T1 signal similar to or greater than that of water to show hyperintensity. This can lead to
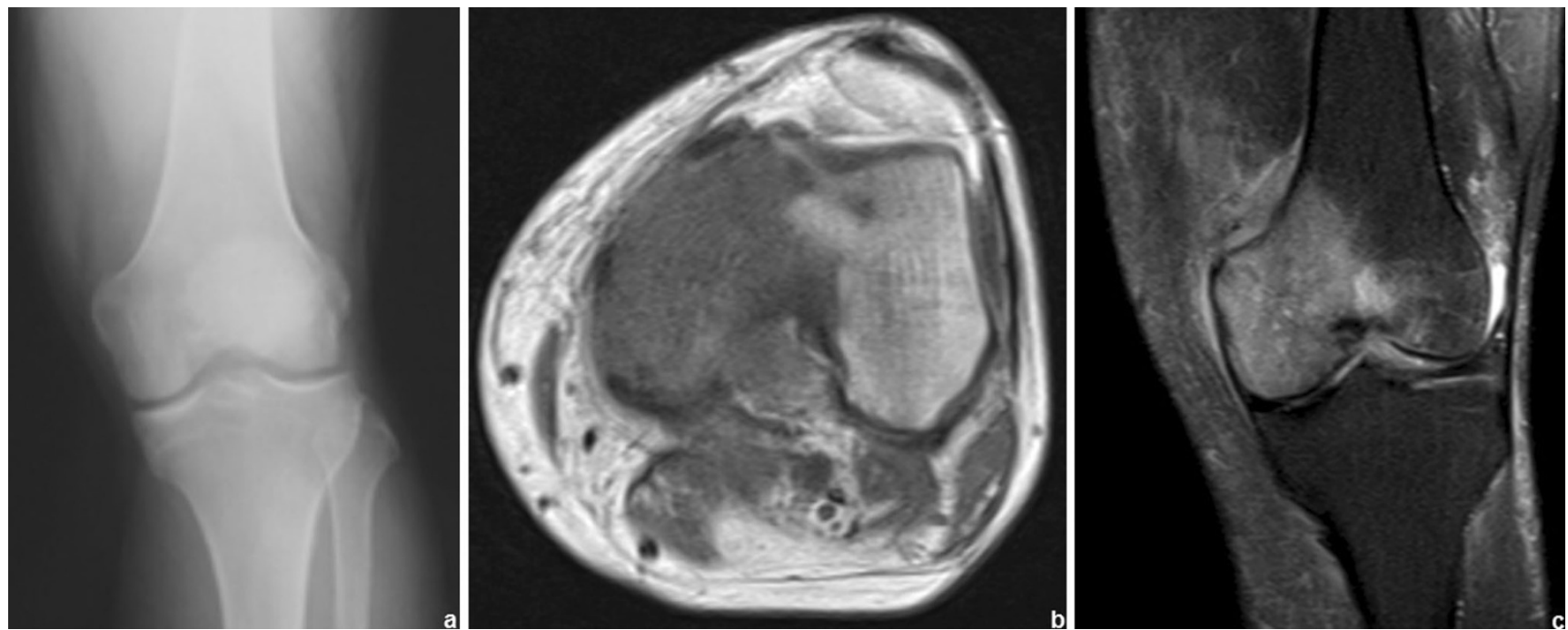

Fig. 4 Patient with chronic left knee pain. Radiographs were obtained (a - normal frontal radiograph). Subsequent MRI study showed abnormal signal intensity in the bone: low T1WI signal (b) and T2FS WI hyperintense lesion (c) related to a bone lymphoma 


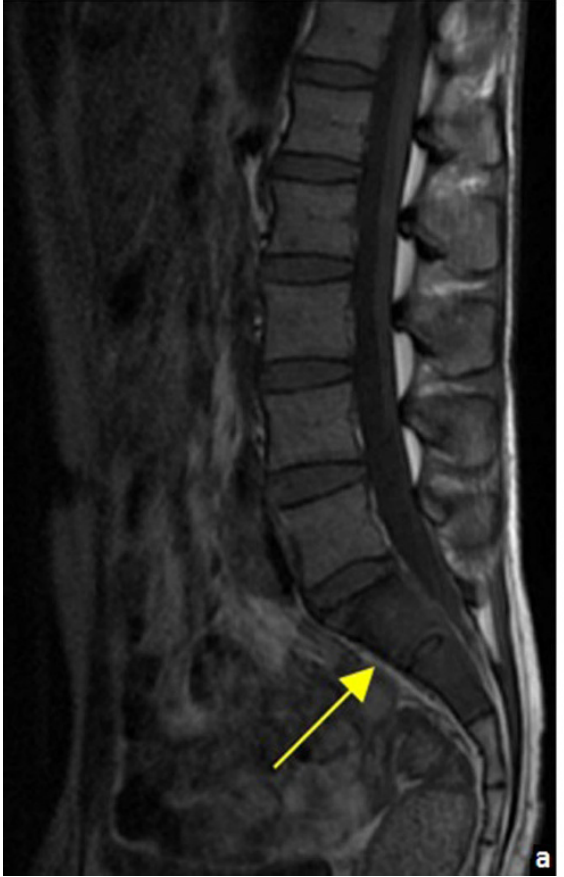

Fig. 5 A 28-year-old patient with a testicular neoplasm and spinal metastases. T1FS WI showed abnormal signal in the superior sacral elements (arrow in a). CEMRI showed diffuse enhancement of the

overestimation of the tumoral extension and compromise its characterisation [4].

\section{Chemical shift imaging}

Chemical shift-based fat suppression can help evaluate bone marrow infiltration [5]: it increases the sensitivity for detection of metastases and myeloma lesions and may be used to improve specificity when equivocal marrow changes are seen
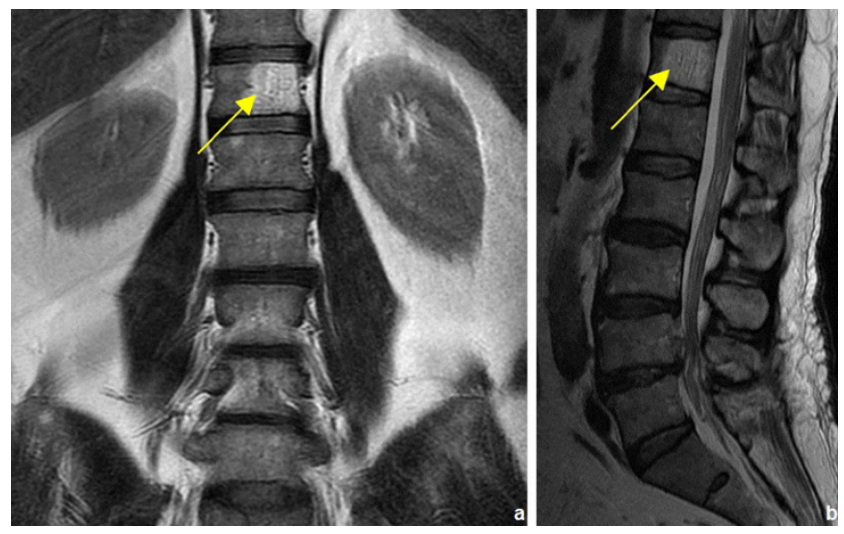

Fig. 6 Coronal T1WI (a) and sagittal T2WI (b) show a wellcircumscribed vertebral lesion, with signal intensity similar to that of the subcutaneous fat: vertebral haemangioma

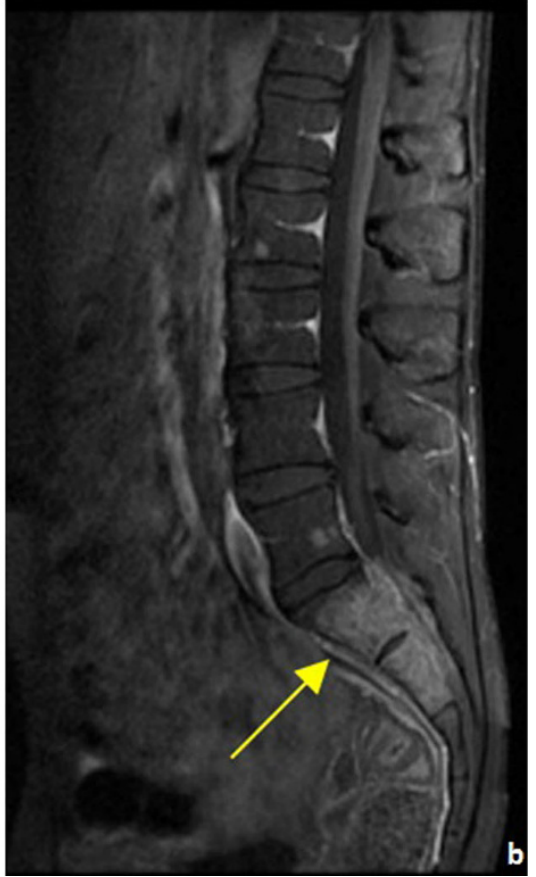

superior sacral elements (arrow in b) with spinal cord extension and focal lesions in the lumbar vertebrae

on MRI [6]. Tumoral tissue will not show signal loss in opposed-phase images, in contrast to normal fatty or haematopoietic red marrow.

Using intravenous gadolinium-based contrast medium

Most bone tumours and tumour-like lesions have a significant amount of cartilaginous tissue (hyperintense on T2WI). CEMRI can be used in the differentiation between solid hyperintense and fluid-containing lesions. Solid, nonnecrotic areas will show diffuse enhancement while liquid will not (Fig. 1).

Gadolinium-based contrast medium also helps distinguish oedema from viable tumour (Fig. 2) and allows an accurate determination of the degree of vascularisation.

Fast acquisition techniques after gadolinium-based contrast medium injection allow contrast-enhanced dynamic imaging. Time to peak, maximum enhancement, slope (degree of enhancement during the first pass), washout rate and the shape of the signal enhancementtime curve can be determined. Contrast-enhanced dynamic imaging is said to be approximately $80 \%$ accurate in differentiating benign tumours from malignant ones [7]. 

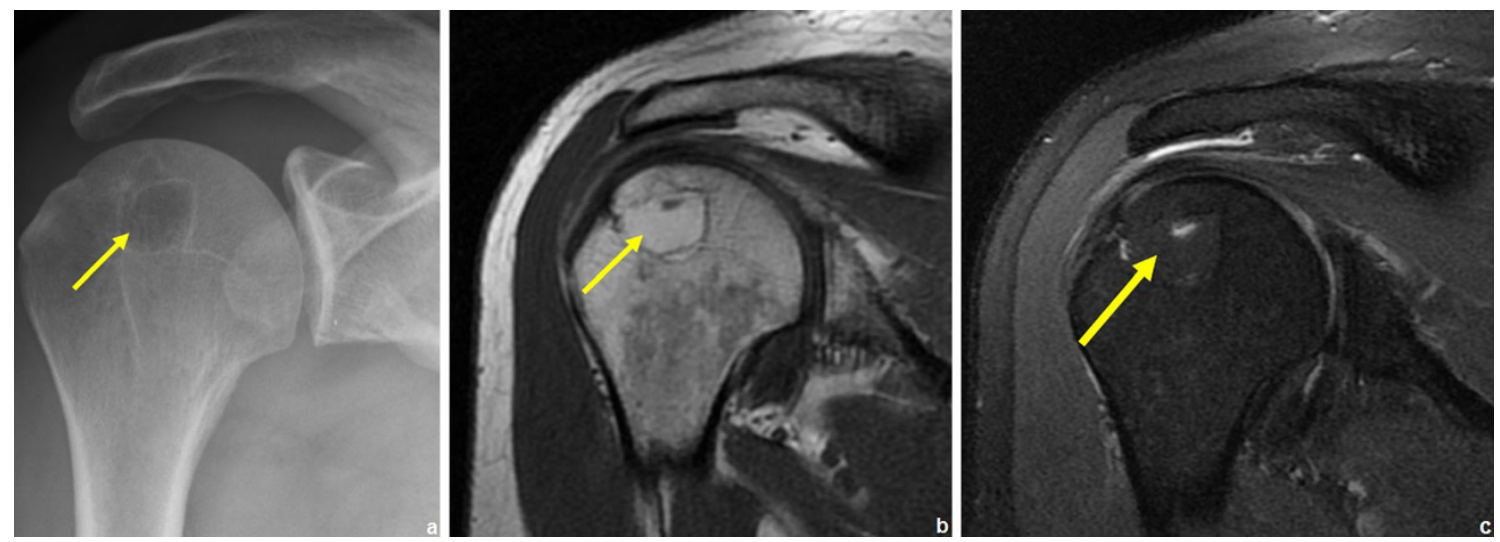

Fig. 7 Patient with a humeral lipoma (arrows in $\mathbf{a}, \mathbf{b}$ and $\mathbf{c}$ ): frontal radiograph shows a lytic lesion in the head of the humerus. This lesion showed signal intensity similar to that of fat on T1WI (b). The signal was suppressed in T2FS WI (c)

Fig. 8 Associated soft tissue component and cortical breakthrough in different cases of Ewing Sarcoma
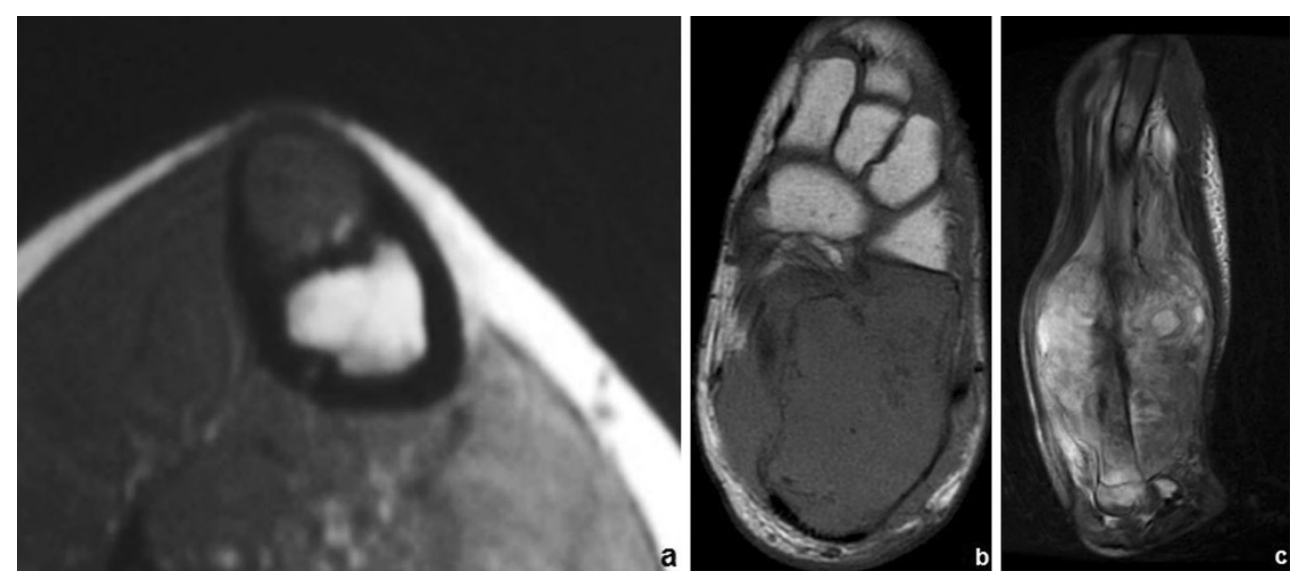

Fig. 9 Typical enchondroma: small lesion located in the distal femur with low/intermediate signal on T1WI and hyperintense on T2WI, containing

calcifications, with no associated soft tissue component or adjacent oedema
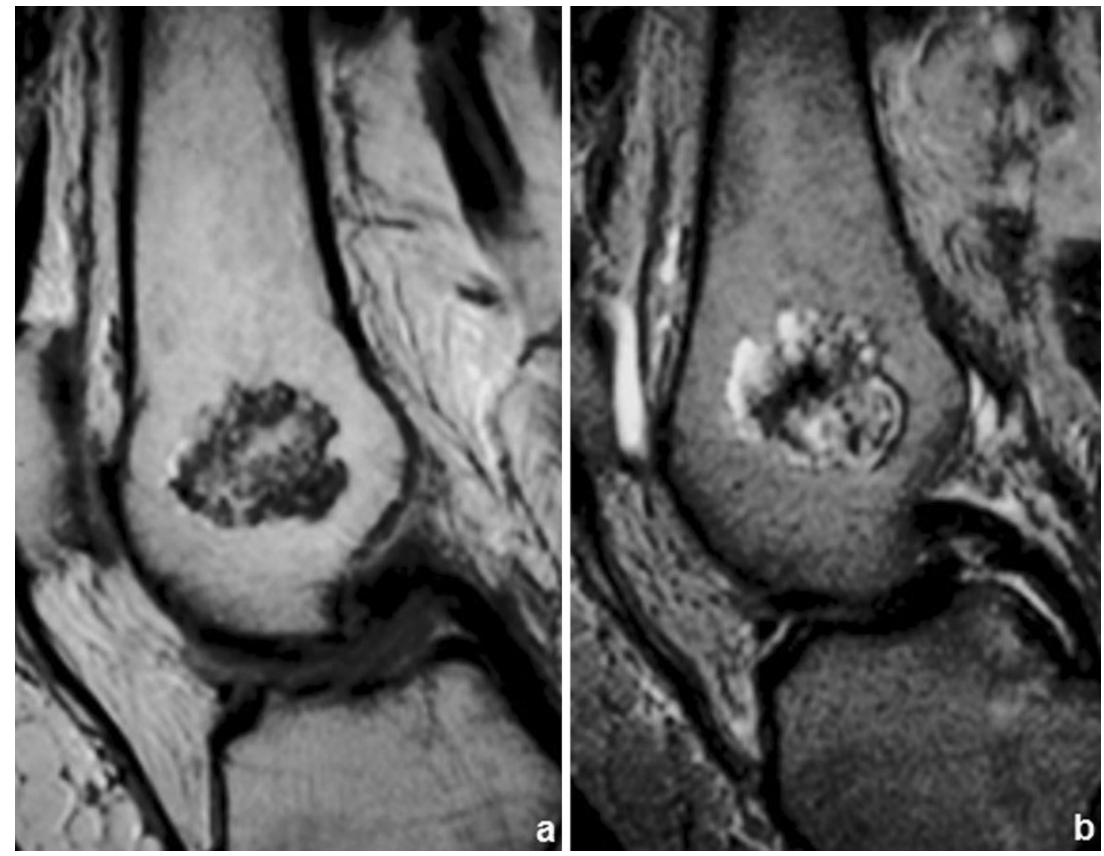


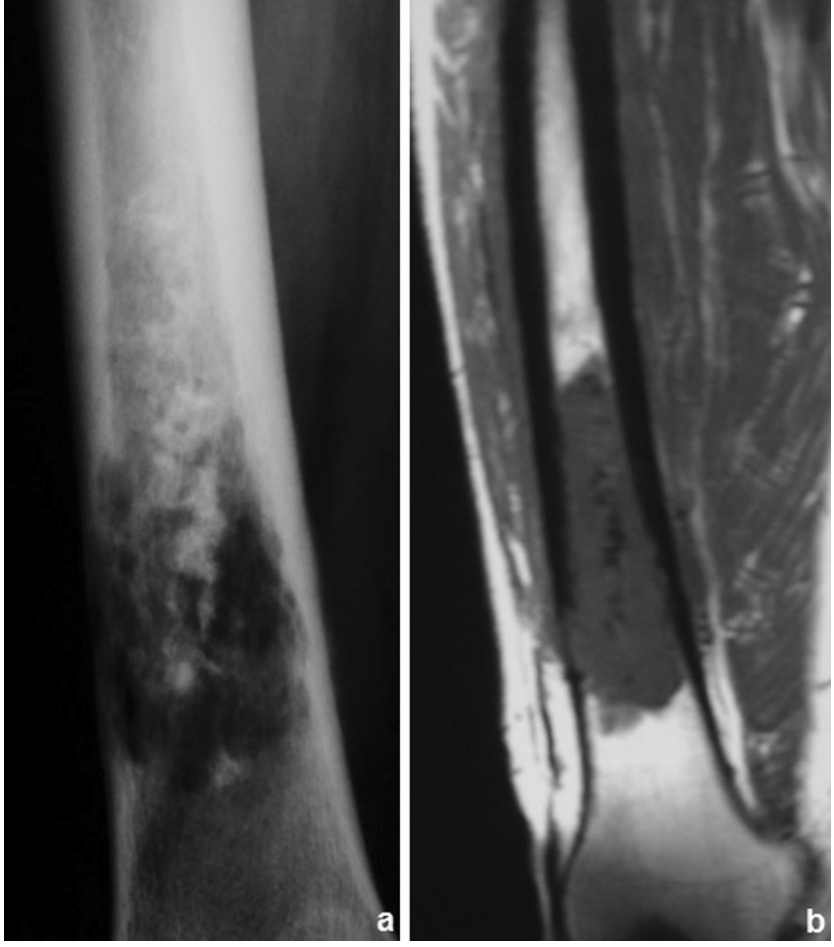

Fig. 10 This patient had chronic lower thigh pain. The radiograph (a) showed an area of bone destruction with central calcification. Coronal T1WI (b) clearly showed a hypointense lesion extending beyond the disrupted cortical tissue. This lesion was a chondrosarcoma

The basic principle that viable active tumoral tissue shows a higher degree of enhancement than nontumoral/ necrotic areas can also be applied to directing biopsies to viable cellular areas and noninvasive assessment of response to therapies such as radiotherapy and chemotherapy. The most reliable indication of necrosis in MRI is probably still simple: a visible lack of enhancement after the administration of gadolinium-based contrast medium.

\section{Diffusion-weighted imaging (DWI)}

DWI shows restriction to the diffusion of water molecules in malignant tumours [4] (Fig. 3). A favourable therapeutic response is associated with a decrease in the signal intensity in high $\mathrm{b}$ values [8]. Apparent diffusion coefficient (ADC) ratios may also be used for assessing the response to neoadjuvant therapy [9]. The use of DWI however is still not included in the guidelines for routine evaluation of malignant tumours [10].

\section{MR spectroscopy imaging}

Wang et al. [11] showed elevation of the choline peak in the majority (18 of 19 patients) of malignant bone
Table 1 List of clinical and imaging features used in the differential diagnosis between enchondroma and chondrosarcoma

\begin{tabular}{ll}
\hline Enchondroma & Chondrosarcoma \\
\hline $\begin{array}{l}\text { More common in extremities } \\
\text { No pain (only if associated with } \\
\text { fracture) }\end{array}$ & $\begin{array}{l}\text { More common in the axial skeleton } \\
\text { Can cause pain }\end{array}$ \\
$\begin{array}{ll}\text { No periostitis } \\
\text { No growth beyond skeletal maturity }\end{array}$ & $\begin{array}{l}\text { Can have associated periostitis } \\
\text { skeletal maturity }\end{array}$ \\
No soft tissue component & Associated focal cortical thickening \\
Absence of bone oedema & Different patterns of signal intensity \\
Size usually $\leq 4 \mathrm{~cm}$ & Bone destruction \\
& Loss of calcifications on follow-up \\
& Soft tissue component \\
& Bone marrow oedema \\
& Size usually $>4 \mathrm{~cm}$
\end{tabular}

tumours and also concluded that spectroscopy can help differentiate malignant from benign tumours by revealing the presence or absence of water-soluble choline metabolites. Further investigation is needed [1], but MR spectroscopy seems to show great potential in differentiating benign from malignant lesions.

Establishing appropriate protocols

Every institution should have established protocols for the MSK imaging evaluation of different anatomic locations (which should include proper imaging planes for the specific region studied).

An important issue relates to the fact that most institutions do not routinely use contrast in general MSK studies (a significant number of lesions are detected incidentally). Some even consider that the routine use of gadolinium in the initial MRI evaluation of a possible primary MSK neoplasm is not justified [12]. We however recommend using CEMRI whenever the radiographs and conventional (T1 and T2FS) MR sequences do not provide an obvious specific benign diagnosis. Even when a specific diagnosis is reached, all lesions raising suspicion for malignancy should still be evaluated after gadolinium-based contrast medium injection in order to optimise the border characterisation and separate oedema from active tumour.

MRI findings should always be correlated with a radiographic study. If there are none available, they should be obtained before giving a final report. 

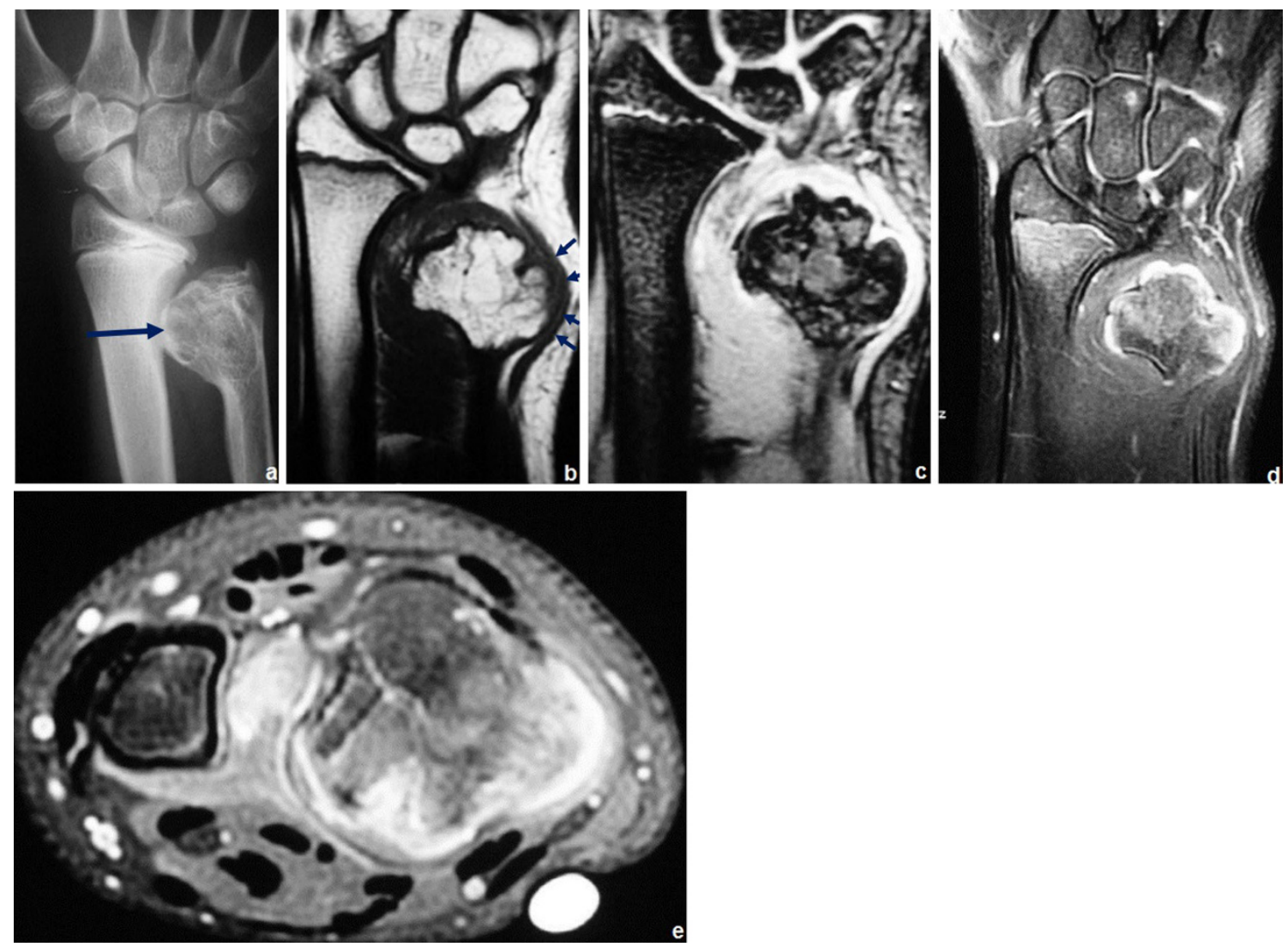

Fig. 11 Anteroposterior radiograph (a) and corresponding MRI study (coronal T1 (b), coronal T2FS (c) and coronal (d) and axial post-Gd T1WI (e) of a broad-base osteochondroma of the ulna. T1WI shows normal bone marrow signal intensity inside the lesion, and T2FSWI shows adjacent effusion and oedema of the adjacent soft tissues. The flexor carpi ulnaris tendon is compressed and displaced (arrows in $\mathbf{b}$ ). The axial post-Gd T1WI (e) shows no compression of the adjacent neurovascular structures

\section{Applying MRI in tumour detection and diagnosis}

\section{(1) Accurate lesion detection}

Faint lytic/sclerotic bone lesions can be difficult to identify using only radiographs. MRI is superior to the other imaging modalities in detecting primary and secondary bone lesions [2] because it can conspicuously show areas of abnormal signal intensity within a bone that should contain normal fatty marrow [13] (Figs. 4 and 5).

In suspected cases of pathological fractures, MRI should be used to evaluate the existence of an underlying lesion [1].

(2) MRI evaluation: aspects favouring benignancy or malignancy

Both benign and malignant tumours usually have inferior signal intensity to the normal marrow signal in T1WI [13]. This is why MRI does not allow accurate prediction of the malignancy or benignancy of a lesion based solely on its signal intensity [13]. There are however some features that, if present, can be highly predictive of a benign etiology, such as the presence of normal fatty marrow [14] or fat within a bone lesion (a common feature in vertebral haemangioma (Fig. 6) and in lipoma (Fig. 7).

MRI can also confirm the presence of fatty or haematopoietic marrow in areas of normally sparse trabeculae (e.g. greater tuberosity of the proximal humerus), which can simulate lytic lesions on radiographs or CT [15].

Typically, benign lesions are well defined and sharply demarcated from the surrounding healthy tissue. Malignant lesions are usually extensive and involve surrounding tissue to a greater extent.

Benign fibrous or expansive lesions can give the false impression of cortical destruction in radiographs (e.g. aneurysmal bone cyst, giant cell tumour and chondroblastoma). The opposite can occur with malignant lesions. MRI can help exclude true cortical destruction (Fig. 8). 


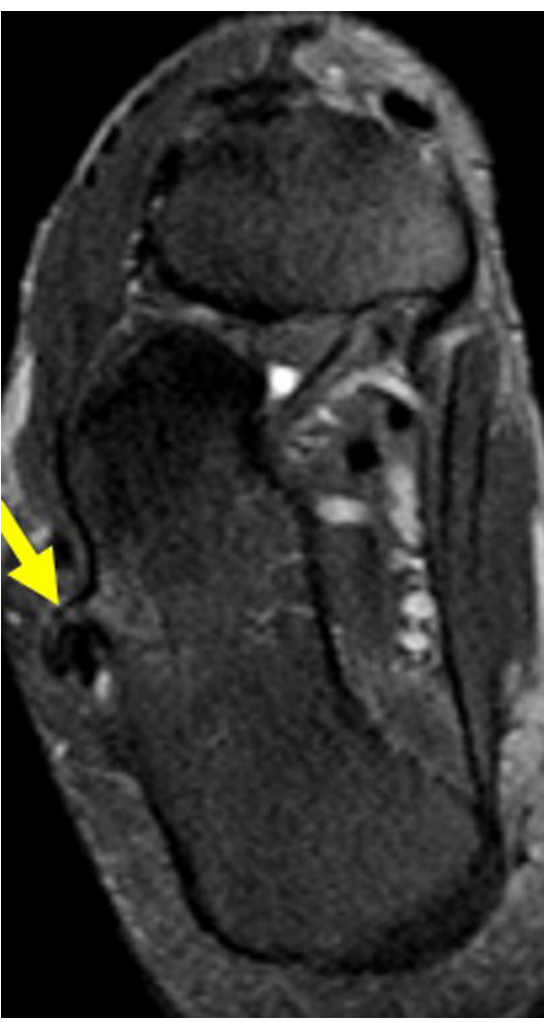

Fig. 12 Young female with ankle pain. This axial T2FSWI shows a small pedunculate osteochondroma of the right calcaneus (arrow) compressing the adjacent fibular tendons

The presence of an associated soft tissue component is one of the most important imaging malignancy predictors in bone tumours. It can point to a malignant degeneration of enchondroma, osteochondroma, previous irradiated areas and Paget's disease. This is usually a prominent feature in Ewing's sarcoma (Fig. 8) and osteosarcoma.

Although osteomyelitis can have an aggressive permeative aspect in radiographs, it usually does not have

Table 2 List of clinical and imaging features used in the differential diagnosis between osteochondroma and secondary chondrosarcoma

\begin{tabular}{lc}
\hline Benign osteochondroma & Secondary chondrosarcoma \\
\hline $\begin{array}{l}\text { No pain (only for fracture, bursitis or } \\
\text { compression of adjacent }\end{array}$ & Can cause pain \\
structures) & \\
$\begin{array}{l}\text { No growth beyond skeletal } \\
\text { maturity }\end{array}$ & $\begin{array}{c}\text { Rapid growth, suspicious especially } \\
\text { if after skeletal maturity }\end{array}$ \\
& Presence of calcifications (signal \\
No associated soft tissue mass & Poids) beyond the stalk \\
& tissue mass \\
Thin cartilagineous cap $(\leq 1 \mathrm{~cm})$ & Thick cartilagineous cap ( $>1 \mathrm{~cm}$ \\
& $[16])$ \\
\hline
\end{tabular}

an associated solid soft-tissue mass [16]: MRI can help distinguish it from true permeative neoplasms such as Ewing's sarcoma.

The absolute value of tumour-associated oedema in the diagnosis is limited, as its degree often does not correlate with the degree of malignancy or tumour aggressiveness [17].

In some cases, differentiating an enchondroma (Fig. 9) from a low-grade chondrosarcoma (Fig. 10) can be a difficult task for both radiologists and pathologists. Many important suspicious imaging features can be assessed using MRI (Table 1).

MRI can also make a difference when evaluating lesions located in the bone surface by determining whether they penetrate the cortical and invade the medullary region [16].

Osteochondromas show an uninterrupted medullary and cortical continuity with the parent bone (Fig. 11). Radiographs often do not allow an accurate evaluation of these features, which is why they should be assessed using CT or MRI. Their presence is helpful in excluding other less common tumours and tumour-like conditions (e.g. osteoma, periosteal chondroma, juxtacortical and soft tissue osteosarcomas and myositis ossificans) [16]. A cartilage cap is often demonstrated, which has low to intermediate SI on T1WI and is hyperintense on T2WI. Several complications can occur in osteochondroma, including osseous deformity, fractures (of the lesion or of adjacent bones), compression of adjacent structures (tendons [Figs. 11 and 12], vessels and nerves), bursitis and rarely [18] malignant degeneration (Table 2; Fig. 13). MRI represents the most valuable imaging modality in symptomatic cases because it can demonstrate typical features of associated soft tissue pathology [18, 19]. The cap thickness is the best predictor of malignant change [17]. The normal upper limit for the cartilagineous cap thickness in MRI is usually considered to be $1 \mathrm{~cm}$ [16]

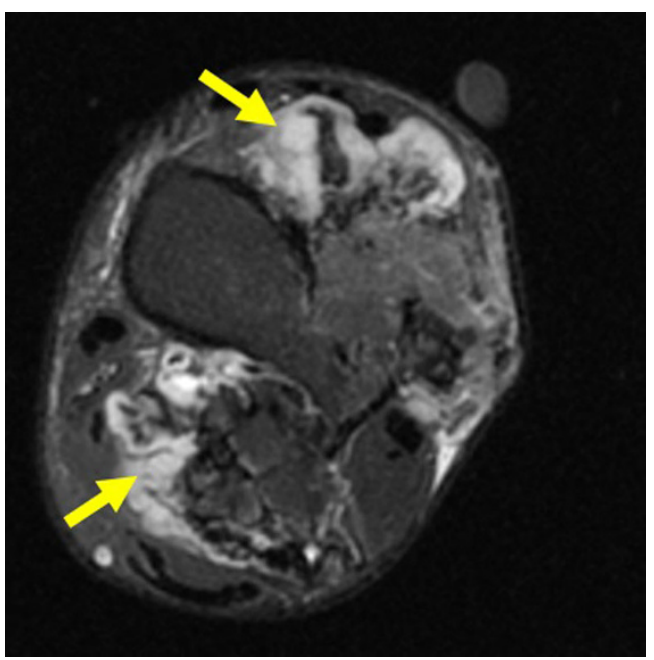

Fig. 13 Axial T2FS WI shows a voluminous lesion with a thick cartilagineous cap: a secondary chondrosarcoma of the left distal tibia 
Fig. $14 \mathrm{ABC}$ of the left femur: coronal (a) and axial (b) T2FS WI: Typical fluid-fluid levels are seen, with no intervening soft tissue or thick septations. The cortical is expanded but not disrupted
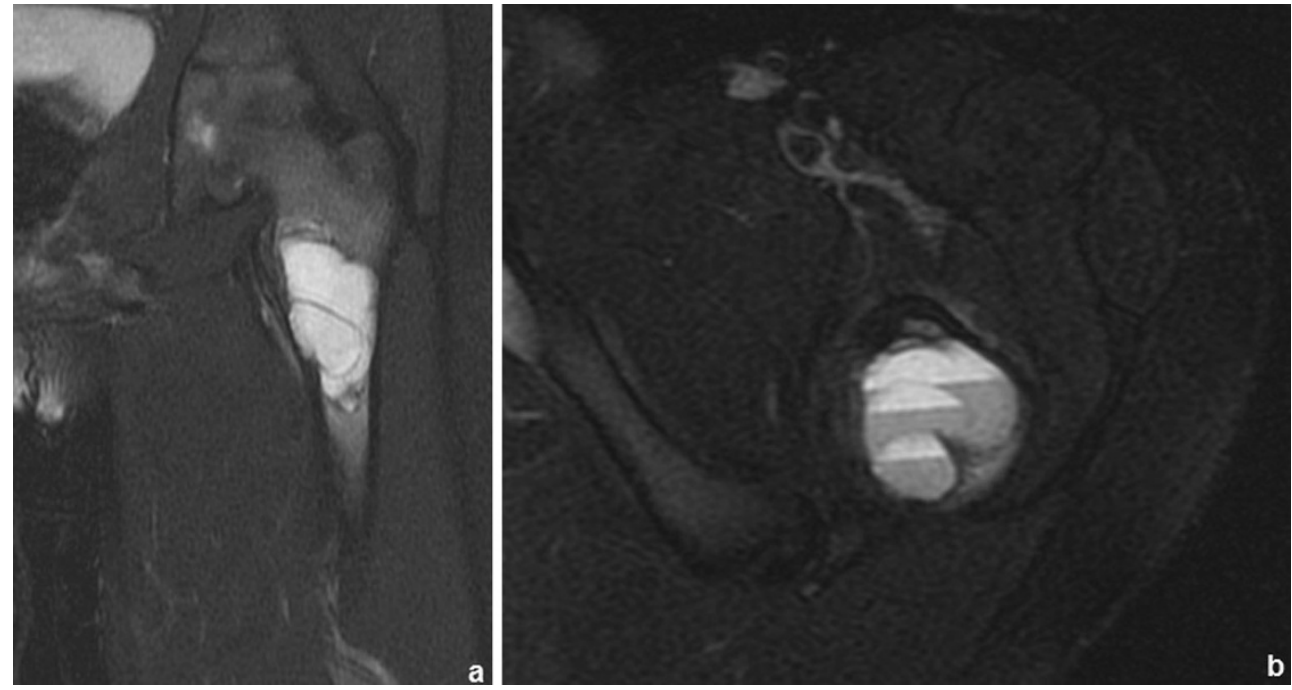

Fig. 15 Axial T2WI (a) and sagittal post-contrast T1WI (b) show typical features of telangiectatic osteosarcoma: a very large large lesion, with a solid component in the posterior dependent part (arrow in a) and extension beyond the disrupted cortical (b)
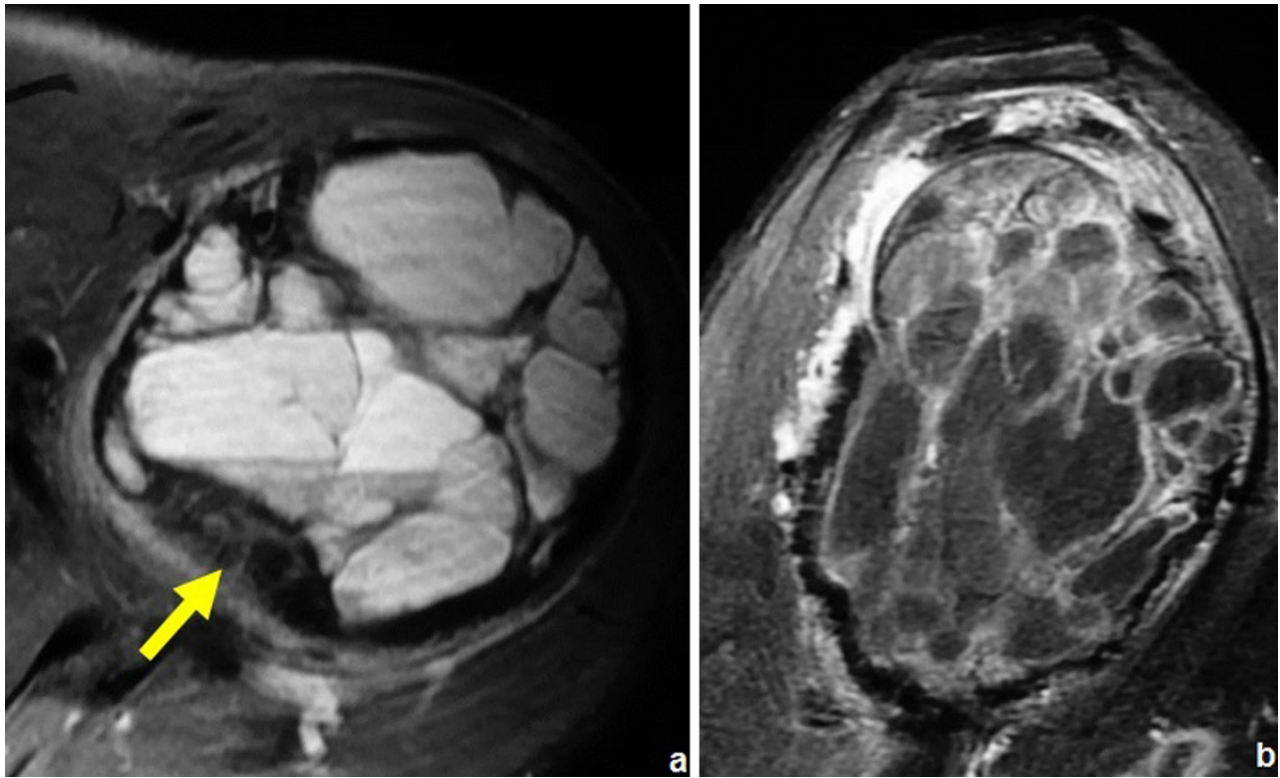

although younger patients in active growth can reach a width of $3 \mathrm{~cm}$ [19].

Flow voids have been demonstrated in vascular tumours (e.g. haemangioma and haemangioendothelioma), renal cell and hepatocellular carcinoma metastases and are uncommon in other tumours [17]. They can therefore be

Table 3 MRI features that favour the diagnosis of telangiectatic osteosarcoma as opposed to $\mathrm{ABC}$

Solid tissue components surrounding or associated with the cystic/ haemorrhagic spaces (better depicted after gadolinium-based contrast medium injection) [24]

Focal solid protrusions through the cortical area or cortical destruction with an associated soft tissue mass [4]

regarded as an additional diagnostic feature but do not have a prominente role in the differentiation of benignancy vs malignancy.

Table 4 Primary bone tumours and tumour-like lesions with low signal T2WI

Giant cell tumour

Chondroblastoma

Natural involution of

some lesions
Predominantly hyperintense in T2WI but, in $63 \%$ of the cases, will show low signal areas that occupy $\geq 20 \%$ of the lesion size [19] (Fig. 16)

$86 \%$ lesions have T2 hypointense areas, entirely or partly [23] (Fig. 17)

Nonossifying fibroma (Fig. 18) 
Fig. 16 Giant cell tumour of the proximal femur in a 36-year-old patient: the coronal T2WI (b) shows a predominantly hyperintense lesion containing areas of low signal intensity; corresponding radiograph in (a)
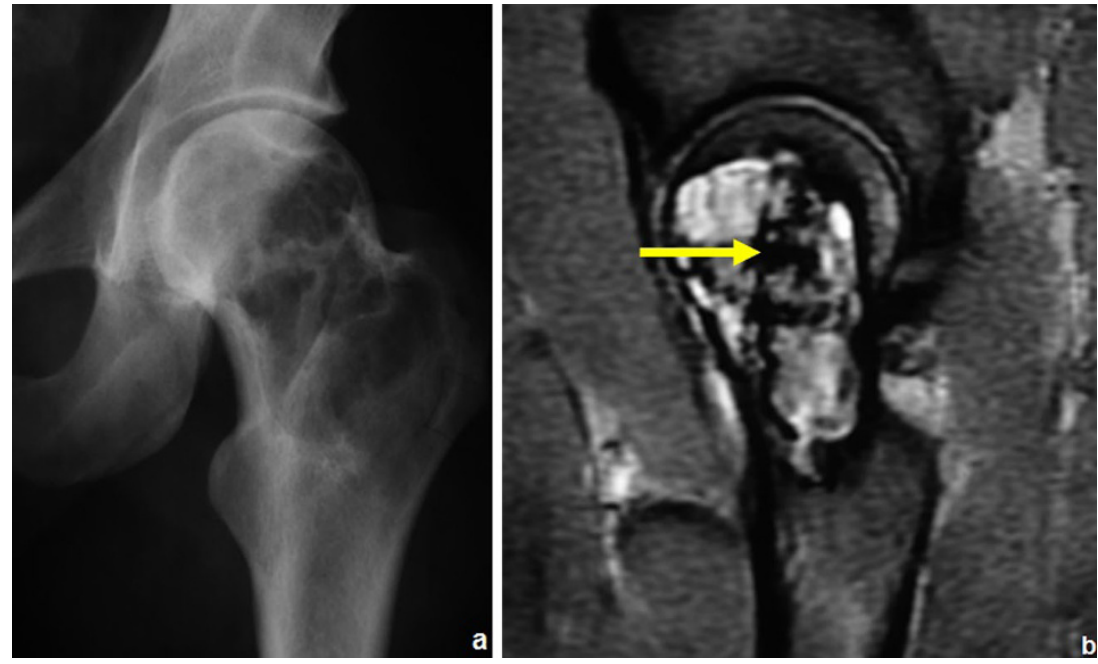

(3) MRI evaluation of fluid-fluid levels (FFLs)

FFLs result from separation of two fluids of differing densities within aneurysmally dilated cavities and generally are most conspicuous on T2WI. They are most common in aneurysmal bone cysts (ABCs) (37-87\%), but are also frequent in osteosarcomas, giant cell tumours and chondroblastomas [20]. They occur less frequently in lesions such as fibrous dysplasia, osteoblastomas, simple bone cysts and brown tumours [20, 21]. FFL cannot be considered diagnostic of any particular type of tumour, and the diagnosis should be made on the basis of other radiological and clinical findings [21]. The most important differential diagnosis of bone lesions with FFLs is ABC (Fig. 14) vs. telangiectatic osteosarcoma (Fig. 15), because while the former is the most common cause of FFLs, the latter is a high-grade malignant lesion (see Table 3 for MRI aspects useful in the differential diagnosis).

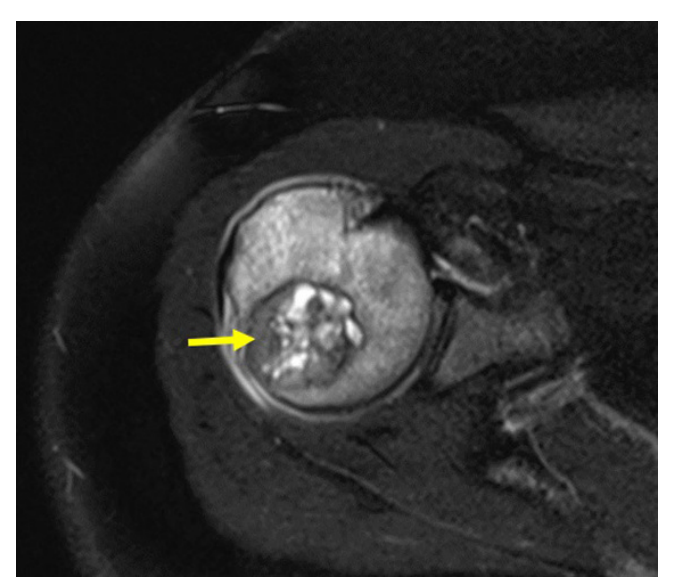

Fig. 17 Axial T2FSWI of a chondroblastoma with partial hypointense content (arrow). There is extensive oedema in the adjacent bone, which is typical of these lesions
(4) Primary lesions with low signal in T2WI

Most bone tumours and tumour-like lesions have a significant chondroid/cartilaginous or liquid component, which is associated with high signal intensity on T2WI. There are however primary lesions that can show partial or entire low signal intensity in T2WI because of an immature chondroid matrix, haemosiderin and calcifications [23]. This feature can be used for making the specific diagnosis (Table 4; Figs. 16, 17and 18).

(5) Evaluation of patients with haematopoietic malignancies and premalignant gammopathies

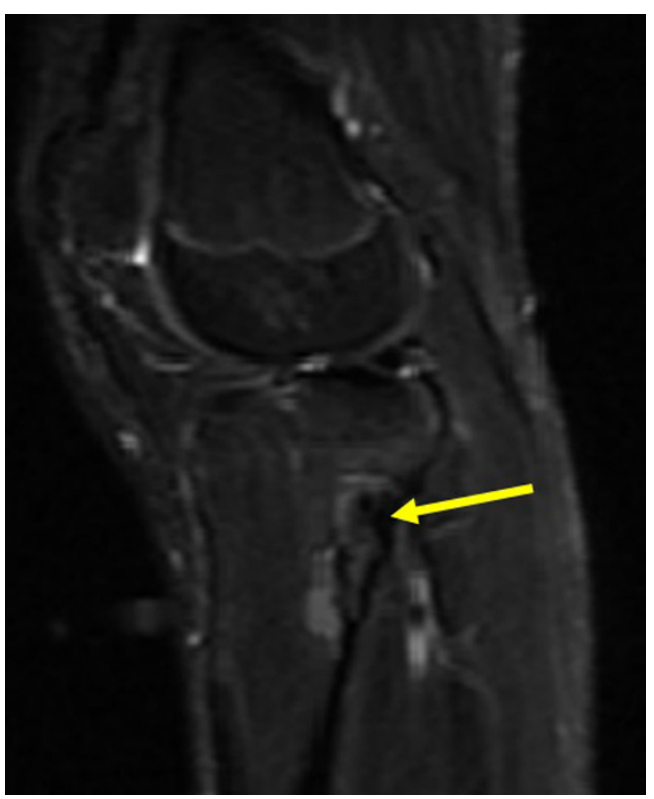

Fig. 18 Sagittal STIR image of the knee shows a partial sclerotic component (low signal intensity) within a nonossifying fibroma 
Table 5 New imaging techniques in the Durie-Salmon PLUS staging system [22]

\begin{tabular}{ll}
\multicolumn{2}{l}{ Durie-Salmon PLUS staging system } \\
\hline Classification & New imaging (MRI and/or FDG PET) \\
\hline MGUS & All negative (normal marrow) \\
Stage IA & Can have single plasmacytoma and/or \\
(Smouldering or indolent) & Limited disease (definition evolving) \\
Multiple myeloma & \\
IB & $<5$ focal lesions; mild diffuse disease \\
IIA/B & $5-20$ focal lesions; moderate diffuse disease \\
IIIA/B & $>20$ focal lesions; severe diffuse disease \\
\hline
\end{tabular}

MGUS: Monoclonal gammopathy of undetermined significance

A: serum creatinine $<2.0 \mathrm{mg} / \mathrm{dl}$; no extramedullary disease (EMD)

B: serum creatinine $>2.0 \mathrm{mg} / \mathrm{dl}$; extramedullary disease (EMD)

Mild difuse disease: micronodular pattern; moderate disease: diffuse low signal intensity on T1WI, but contrast between the bone marrow and disk remains; severe disease: contrast between bone marrow and disk is lost or inverted (bone marrow shows a signal intensity equal or inferior to that of the disk on T1WI)

For patients with Hodgkin's lymphoma and high-grade non-Hodgkin's lymphoma, FDG-PET has been established as the imaging modality of choice for staging and monitoring treatment. MRI, however, should be considered in cases with high risk of bone marrow involvement and equivocal findings on PET or extracompartmental tumour growth as well as in patients with potential treatment complications [24].

MRI is a noninvasive technique that can complement bone marrow aspirations (the iliac crest biopsy may be false negative when bone marrow infiltration is focal rather than diffuse) [25].

Radiologic skeletal surveys are still part of the recommended baseline evaluation in multiple myeloma (MM), despite their limitations (they only depict lytic lesions after the loss of over $30 \%$ of the bone mineral density, poorly show anatomic areas such as the ribs, pelvis and spine and are not accurate for the evaluation of diffuse medullary extension) [26]. In several studies, MRI showed a higher sensitivity than skeletal surveys for the detection of focal bone marrow lesions [27, 28]. MRI's increased diagnostic accuracy led to revising the traditional Durie-Salmon staging system (which only included radiographs in the imaging approach). The newer Durie-Salmon PLUS staging system includes MRI in the evaluation of the spine (summarised in Table 5). Staging and treatment can potentially change in 15$25 \%$ of patients [29].

The possibility of extending the spinal MRI evaluation to a whole-body MRI (WBMRI) evaluation of the skeleton in MM is under active investigation because a significant percentage of lesions occur outside the axial skeleton [30].
MRI is now widely used in MM not only for assessing the bulk of disease in patients with osteopaenia or uncertain staging (Fig. 19), but also to document the extent of bone marrow infiltration and cord or root compression in patients with pain syndromes [31]. It may also be used to select appropriate biopsy sites [25].

Monoclonal gammopathy of undetermined significance (MGUS) and smouldering multiple myeloma (SMM) are asymptomatic, pre-malignant disorders that can progress to MM. The Durie-Salmon PLUS staging system includes features that allow a clear distinction between MGUS and MM. The MRI findings in SMM are still currently under investigation [8]. In patients with asymptomatic or smoldering myeloma, MRI findings have shown a correlation with the likelihood of transition to MM [29].

Patients with MGUS and SMM require indefinite followup given their lifelong risk of progression to MM or related malignancy [32]. A skeletal survey should be repeated at least once every year for SMM [32], but MRI may be better suited for the imaging follow-up.

Despite the improvements that MRI brought to the imaging evaluation of haematopoietic malignancies, there are still limitations regarding sensitivity and specificity. An infiltration with less than $20 \%$ neoplastic cells cannot be distinguished from normal marrow with standard MR pulse sequences [24]. Several authors reported a normal bone marrow MR signal in patients with leukaemia, in patients with early stages of bone marrow invasion by lymphoproliferative diseases and even in up to one-quarter of patients with stage III MM [24, 33]. MRI specificity of signal alterations of bone marrow is low, which is why the findings need to be correlated with clinical and laboratory findings.

Coronal T1 and STIR images of the entire skeleton (WBMRI) can be acquired quickly and provide good morphological evaluation of the extent of the disease. Functional imaging with contrast-enhanced dynamic imaging can provide information regarding disease activity [34]. The future role of these techniques in oncohaematological diseases still needs to be defined, but WBMRI will probably become the bone marrow imaging method of choice.

\section{Staging malignant bone tumours using MRI}

The American Joint Committee on Cancer (AJCC) staging system (Table 6) is currently the most used for malignant bone tumours. It separates stages I and II according to the tumour size (whether $\leq$ or $>8 \mathrm{~cm}$ ) and defines stage III 

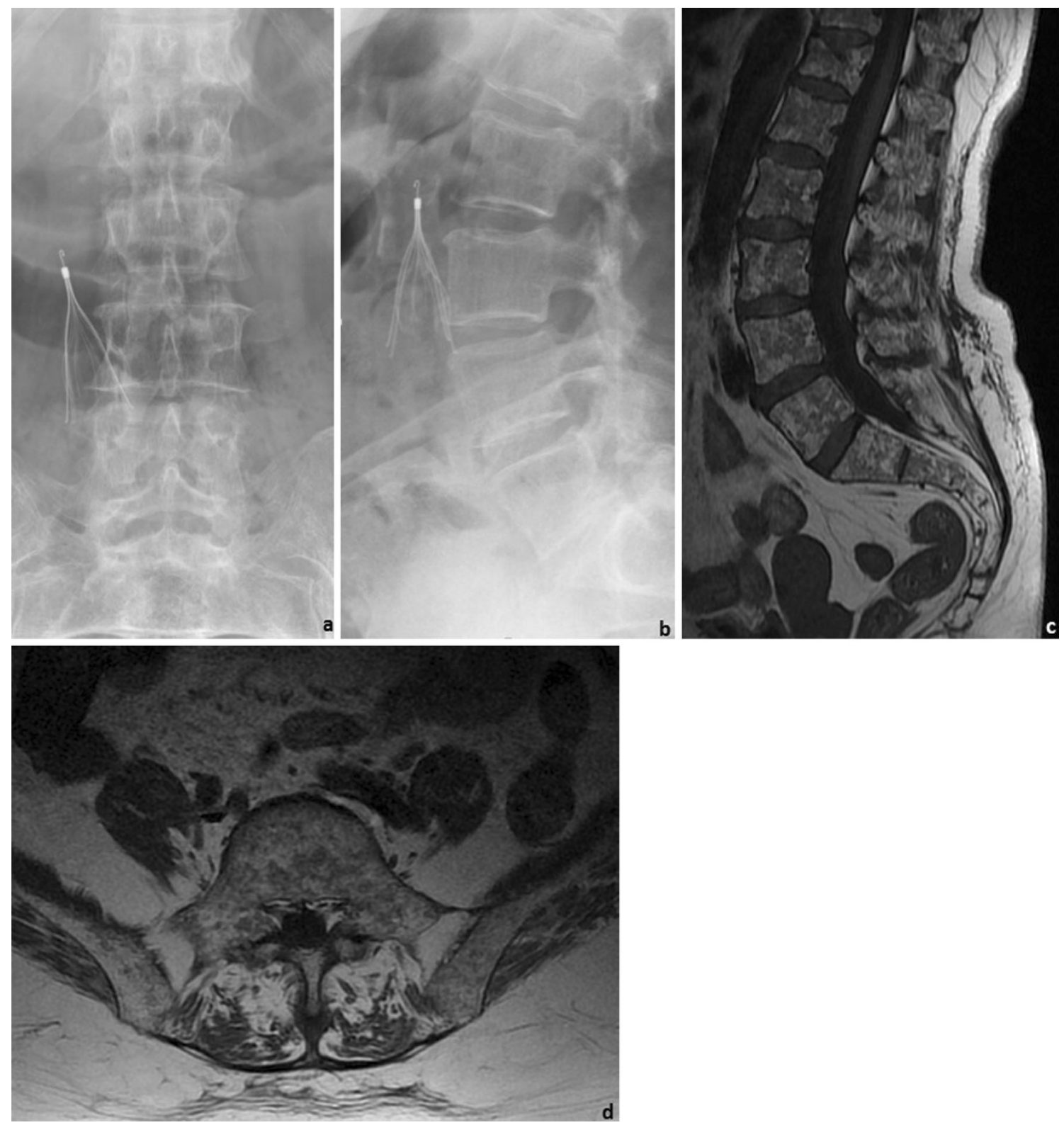

Fig. 19 A 76-year-old female patient with MM: Frontal (a) and lateral (b) radiographs of the lumbar spine and sagittal (c) and axial (d) T1WI of the lumbosacral spine. The radiographic study showed diffuse osteopaenia of the lumbar vertebrae, which could solely be related to

for cases with "skip metastasis" (discontinuous tumours in the primary bone site). Stage IV is subdivided according to the presence or absence of metastases in locations other than the lung.

\section{Local staging and preoperative assessment}

Both CT and MRI can provide preoperative planning and staging, but existing data suggest that MRI should be the preferred technique $[1,35]$. primary osteoporosis given the patients' gender and age. The MRI study however showed innumerous focal lesions, consistent with the diagnosis of MM. Note: The small cone-shaped device present in the radiographs is an inferior vena cava filter
T1WI and CEMRI can accurately depict the extension of the primary lesion. Its exact location should be clearly stated in the report (e.g. measuring the distance of the lesion to an anatomic reference will help planning surgical procedures).

The distinction between tumour and oedema can be difficult, but is essential for local staging and guiding biopsy. Tumoral tissue has a more heterogeneous signal than that of associated oedema. Contrast-enhanced dynamic imaging can also be used (the oedema will show a slope $20 \%$ or less than the tumour itself) [17]. 
Table 6 AJCC staging system (adapted from the AJCC Cancer Staging Forms, 7th Edition)

American Joint Committee on Cancer(AJCC) staging system

\begin{tabular}{lllll}
\hline Stage & Primary tumour (T) & Regional lymph nodes (N) & Distant metastasis (M) & Histologic grade (G) \\
Stage IA & T1 & N0 & M0 & G1, 2 (low grade) \\
Stage IB & T2 & N0 & M0 & G1, 2 (low grade) \\
& T3 & N0 & N0 & G3, 4 (high grade) \\
Stage IIA & T1 & N0 & M0 & G3, 4 (high grade) \\
Stage IIB & T2 & N0 & M0 & G3, 4 (high grade) \\
Stage III & T3 & N0 & M0 & Any G \\
Stage IVA & Any T & N1 & M1a & Any G \\
Stage IVB & Any T & Any N & Any M & Any G \\
& Any T & & M1b & \\
\hline
\end{tabular}

Osteosarcoma and Ewing's tumour are more common in paediatric ages. Though the physeal plate was traditionally thought to be a significant barrier for tumoral spread, it is now known that $75-88 \%$ of osteosarcomas show extension to the epiphysis in children [19]. Physeal and epiphyseal involvement (Fig. 20) need to be assessed in order to determine whether joint-sparing surgery can be performed. This should be done using T1WI and T2FS WI/STIR. Abnormal physeal signal intensity, especially when contiguous with and isointense to the primary tumour, is the most important feature [4].

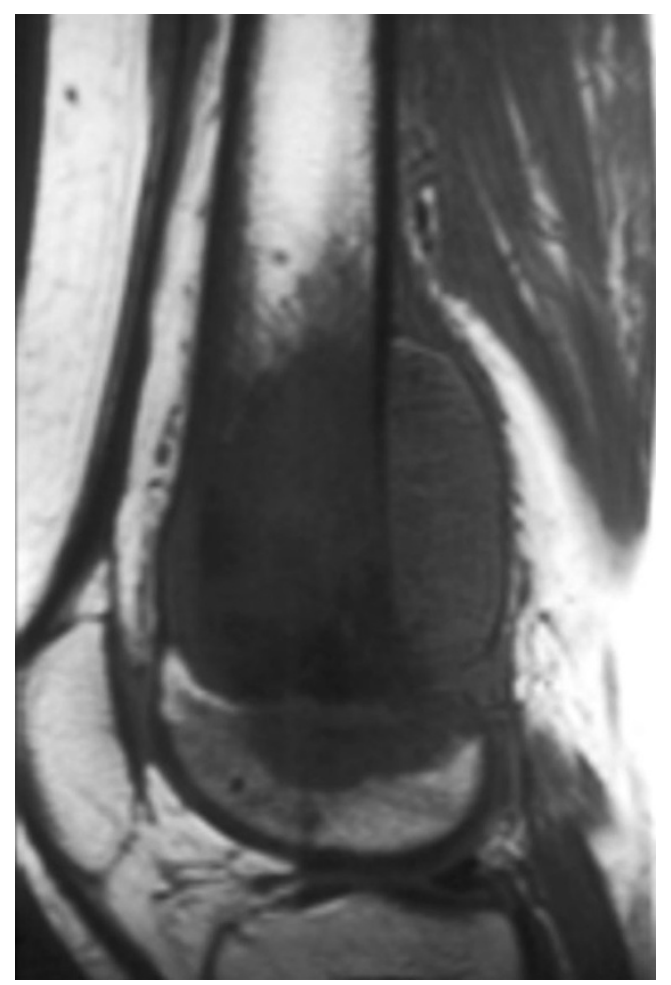

Fig. 20 T1W sagittal image of an osteosarcoma of the distal femur showing extension beyond the physeal plate
Direct visualisation of a tumour extending into the articular cavity through a destroyed cortical region is the most obvious sign of joint involvement (Figs. 21 and 22). Joint effusion alone is not sufficient for its diagnosis but its absence can help exclude it with a high degree of certainty [4].

Muscular invasion should preferentially be assessed in the axial plane using T1WI and T2FS/PD FS WI [4].

MRI is superior to CT and conventional angiography in the evaluation of neurovascular involvement [4]. The

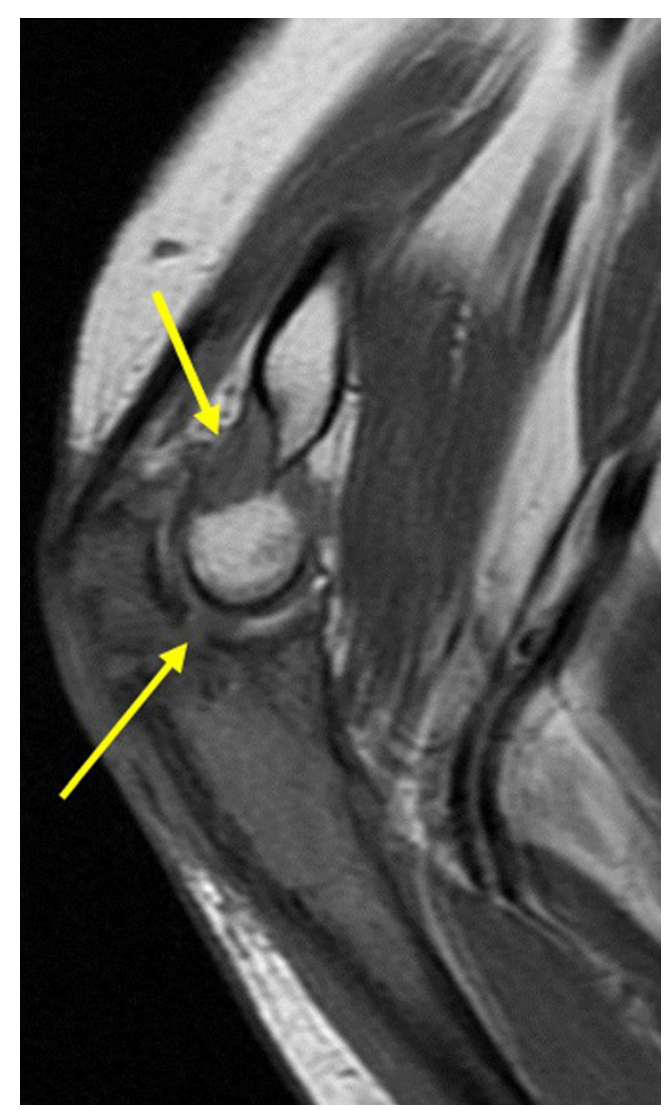

Fig. 21 T1W coronal image of an osteosarcoma of the ulna showing extension into the elbow joint with an associated effusion (arrows) 


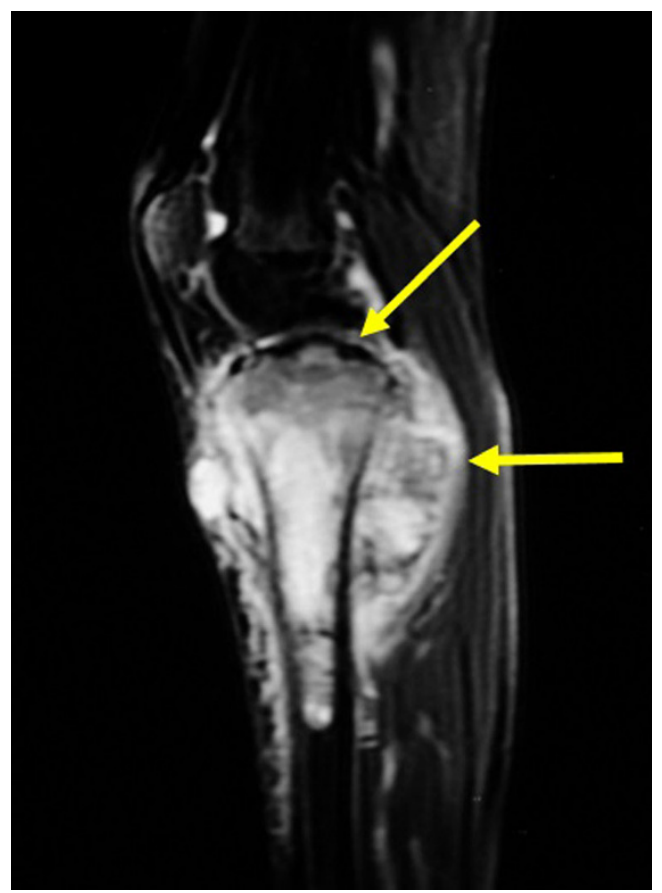

Fig. 22 T2FS sagittal image of an osteosarcoma of the proximal tibia: there is muscular invasion (lower arrow) and intra-articular extension (upper arrow)

best predictors are loss of the perivascular/perineural fat and encasement (especially with associated stenosis). They should be assessed in the axial plane using T2FS/PD FSWI and CEMRI (Fig. 23).

\section{Use of MRI in evaluating distant extension}

Skip metastases (Fig. 24) are considered rare (less than $5 \%$ of osteosarcomas [19]); however, their presence automatically determines an AJCC stage $\geq$ III and is considered a poor prognostic sign [36].

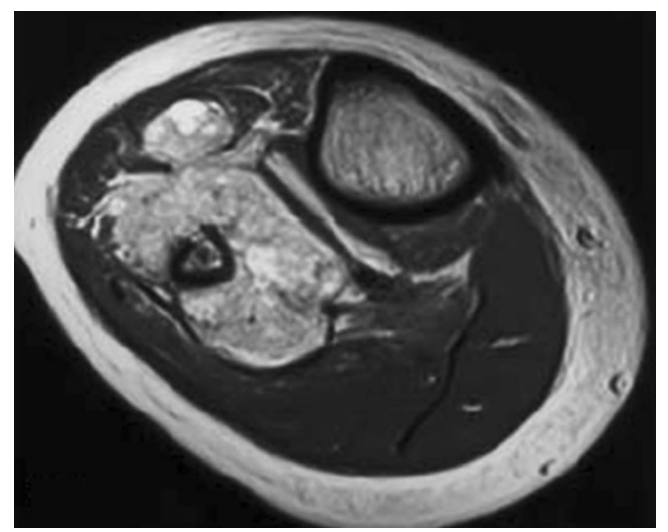

Fig. 23 Gadolinium-based contrast medium-enhanced T1W axial image: osteosarcoma of the right tibia invading the adjacent soft tissues (including the vasculature)

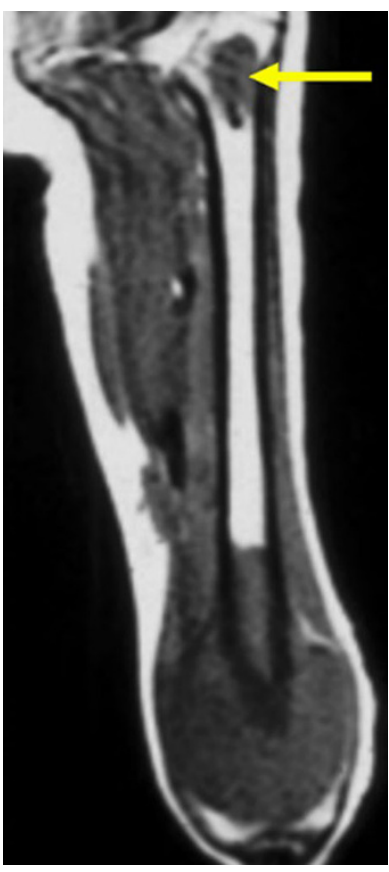

Fig. 24 Coronal T1 image of an osteosarcoma of the distal femur with an associated proximal skip lesion (arrow)

Transarticular skips should be considered stage IV disease (distant metastasis) because aggressive chemotheraphy (used for typical skip metastases, stage III) does not improve diseased-free survival in these patients [37].

MRI has no role in assessing lymph node or lung involvement. It is currently under active investigation as an alternative to bone scan or FDG-PET in the assessment of bony metastasis.

\section{Assessing response to neoadjuvant therapy with MRI}

Chemotherapy has an important role in the treatment of MM, osteosarcoma, Ewing sarcoma's and lymphoma.

Most bone tumours are resistant to radiotherapy [28]: its role is limited to local treatment of Ewing's sarcoma [38], some cases of conventional osteosarcoma (when marginal resection is required for functionality [19]) and chordoma [16].

Chondrosarcomas and malignant fibrous histiocytoma are usually resistant to both chemotherapy and radiotherapy $[16$, 38].

Expected post-radiation MRI changes in successful cases are a decrease in tumour size and an increase in its T2 signal intensity (due to fatty transformation of the bone marrow). 
Fig. 25 Ewing's sarcoma before (a) and after (b) chemotherapy: a decrease in the size of the softtissue component and presence of a circumferential hypointense rim are features suggestive of good response
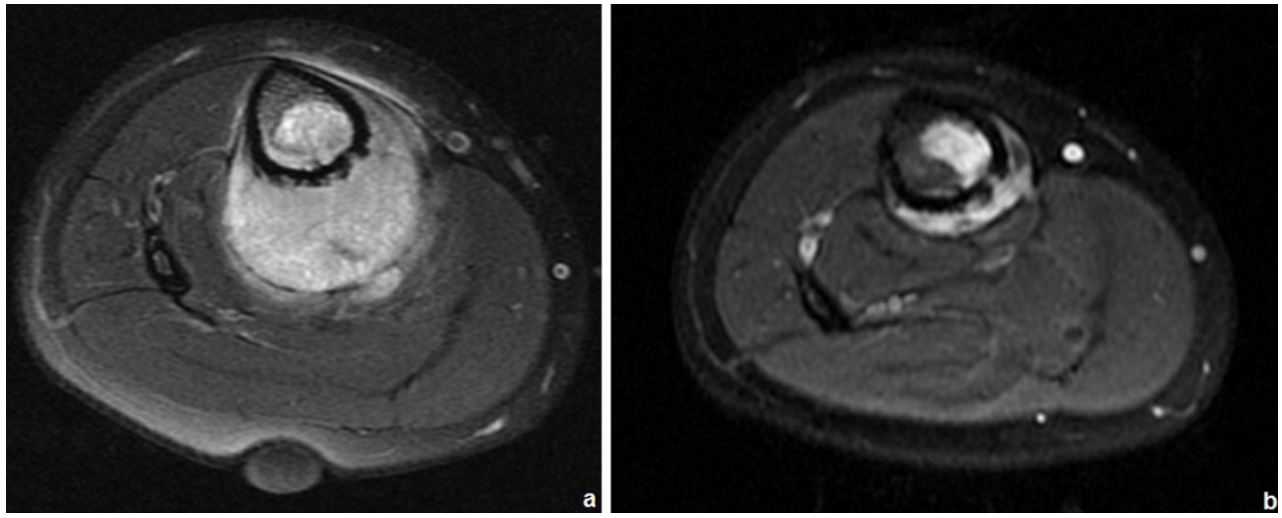
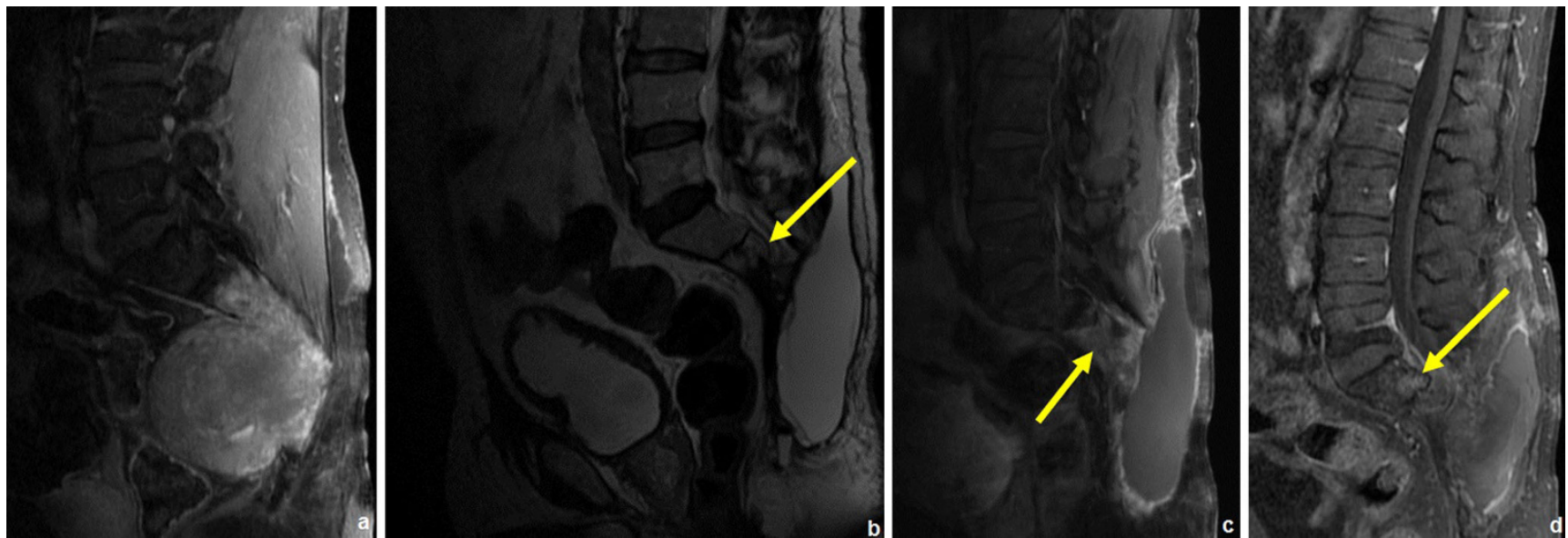

Fig. 26 MRI applied in the diagnosis and follow-up of a patient with chordoma: this patient had a voluminous lesion destroying the distal part of the sacrum (a). He had radiotherapy and surgery and was re-evaluated 4 months later: MRI showed a posterior subcutaneous fluid collection and a localised area with high T2WI signal (arrow in b) and contrast enhancement (arrow in c) in the inferior spine, which could be due to either residual tumoral tissue or radiotherapy. Posterior follow-up however showed a localised round area of persistant enhancement, which proved to be local recurrent/residual tumour (arrow in d)
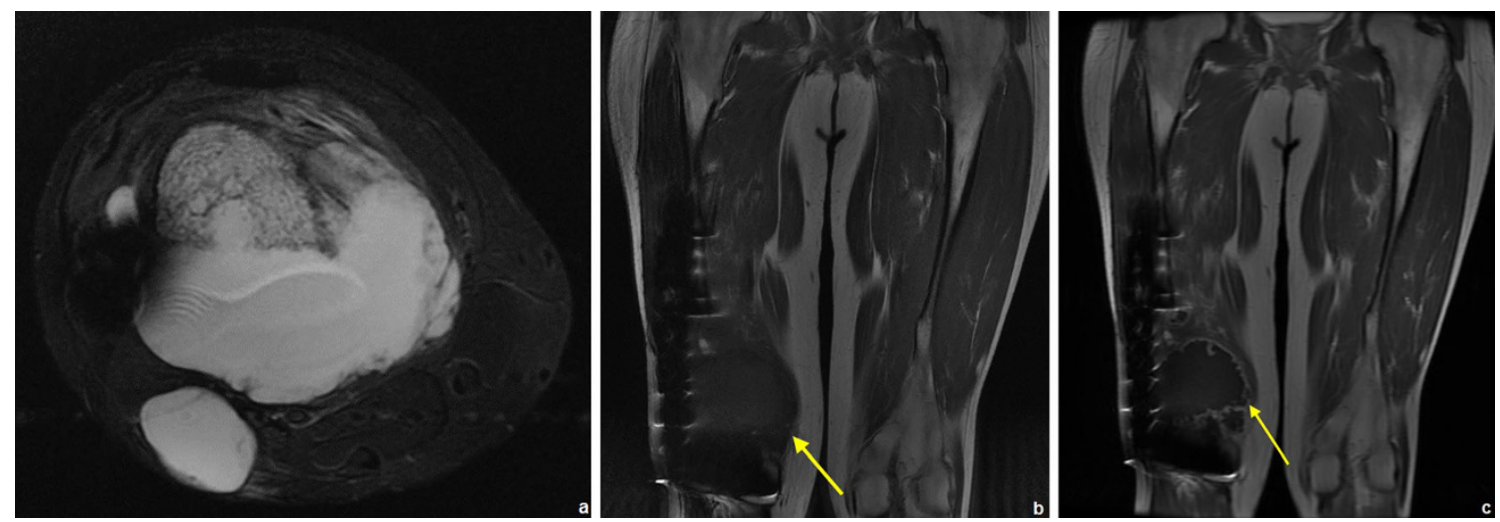

Fig. 27 This patient had resection of the distal femur due to a chondrosarcoma. MRI showed a suspicious area of high signal near the distal prosthesis (a and arrow in b), which showed peripheral enhancing lobulations (arrow in $\mathbf{c}$ ): a local recurrence of chondrosarcoma 
Table 7 Mimickers

\begin{tabular}{|c|c|c|}
\hline Condition & Explanation & Distinguishing features/recommendations \\
\hline $\begin{array}{l}\text { Osteomyelitis } \\
\text { (Figs. } 28 \text { and 29) }\end{array}$ & Aggressive aspect & $\begin{array}{l}\text { Usually involves the metaphysis in children } \\
\text { Small/absent soft tissue component } \\
\text { Penumbra sign* } \\
\text { Fistulous tracts } \\
\text { Clinical features** }\end{array}$ \\
\hline Eosinophilic granuloma (EG) & Aggressive aspect & $\begin{array}{l}\text { Can be very difficult or even impossible to } \\
\text { distinguish from malignant lesions in young } \\
\text { patients } \\
\text { EG of the spine shows preservation of terminal } \\
\text { end plates, disks and posterior elements } \\
\text { (Fig. 30) }\end{array}$ \\
\hline $\begin{array}{l}\text { Stress lesions } \\
\text { (Figs. } 31 \text { and } 32 \text { ) }\end{array}$ & $\begin{array}{l}\text { Elderly patients with insufficiency fractures } \\
\text { Fatigue fractures }\end{array}$ & $\begin{array}{l}\text { Hypointense line extending from the } \\
\text { cortex into the medullary area on } \\
\text { T1- and T2WI } \\
\text { Surrounding oedema [15] } \\
\text { Absence of focal lesion or soft tissue mass [15] }\end{array}$ \\
\hline Bone infarcts and osteonecrosis & $\begin{array}{l}\text { Early osteonecrosis may result in a poorly } \\
\text { defined region of lucency simulating a tumour } \\
\text { in radiographs } \\
\text { Calcifications can simulate those of } \\
\text { chondroid lesions }\end{array}$ & $\begin{array}{l}\text { Usually manifests as a well-defined linear } \\
\text { serpentine rim of low signal intensity on } \\
\text { T1WI. On T2WI the rim may have low } \\
\text { signal intensity, high signal intensity or } \\
\text { both ("double line" sign) } \\
\text { Chondroid lesions show peripheral lobulations } \\
\text { with T2 hyperintensity }\end{array}$ \\
\hline $\begin{array}{l}\text { Myositis ossificans } \\
\text { (Fig. 33) }\end{array}$ & $\begin{array}{l}\text { Can have a disorganised amorphous bone } \\
\text { formation similar to osteosarcoma }[19,44]\end{array}$ & $\begin{array}{l}\text { Usually separated from the cortex } \\
\text { Evolves to mature ossification }\end{array}$ \\
\hline $\begin{array}{l}\text { Haematopoietic marrow } \\
\text { (Fig. 34) }\end{array}$ & $\begin{array}{l}\text { Axial skeleton, thoracic grid, pelvis and } \\
\text { extremities of long bones can maintain areas of } \\
\text { haematopoietic marrow even after skeletal } \\
\text { maturity [15] (this can simulate marrow } \\
\text { infiltration in T1WI and cause high signal } \\
\text { on STIR) }\end{array}$ & $\begin{array}{l}\text { Signal intensity }>\text { than that of muscle on T1WI } \\
\text { Presence of microscopic fat ( }>50 \% \text { drop in } \\
\text { signal in opposed-phase) [15] }\end{array}$ \\
\hline Aggressive osteoporosis & $\begin{array}{l}\text { Sudden immobilisation can cause } \\
\text { bone demineralisation, with oedema } \\
\text { mimicking diffuse tumoral infiltration }\end{array}$ & $\begin{array}{l}\text { Preferential locations: subchondral bone, } \\
\text { tendon and ligamentar insertions }\end{array}$ \\
\hline
\end{tabular}

*The "penumbra sign" on MRI is a rim with higher signal intensity than that of the main abscess on T1WI. It is helpful in distinguishing between subacute osteomyelitis from other osseous lesions [45]

$* *$ Rapid onset of fever, localised pain and oedema; $50 \%$ of cases show positive blood cultures

Table 8 Pitfalls

\begin{tabular}{|c|c|c|}
\hline Pitfall & Explanation & Distinguishing features/ recommendations \\
\hline Very infiltrative lesions (e.g. MM) & $\begin{array}{l}\text { Can preserve bone marrow fat [15] (normal } \\
\text { signal on T1WI and signal drop in out of } \\
\text { phase) }\end{array}$ & Can be undetectable \\
\hline $\begin{array}{l}\text { Anaemia, rebound following } \\
\text { chemotherapy or treatment with } \\
\text { colony-stimulating factor } \\
\text { (Figs. } 35 \text { and } 36 \text { ) }\end{array}$ & $\begin{array}{l}\text { Marked haematopoiesis increases the amount } \\
\text { of red marrow, resembling recurrent } \\
\text { tumoral disease }\end{array}$ & $\begin{array}{l}\text { Usually bilateral and symmetric } \\
\text { Signal intensity }>\text { than that of muscle on T1WI } \\
\text { USPIO-enhanced MRI can differentiate these from tumour } \\
\text { deposits (RES cells are present in the reconverted marrow bu } \\
\text { not present/substantially reduced in tumour deposits [24] }\end{array}$ \\
\hline $\begin{array}{l}\text { Post-chemotherapy osteosarcoma } \\
\text { "size increase" }\end{array}$ & $\begin{array}{l}\text { Sometimes even in "good responders" the } \\
\text { primary lesion does not diminish in size or } \\
\text { appears to enlarge }\end{array}$ & $\begin{array}{l}\text { Therapy has low impact on the mineralised matrix of osteogenic } \\
\text { sarcoma } \\
\text { Matrix maturation/ossification (presence of fatty marrow) }\end{array}$ \\
\hline $\begin{array}{l}\text { Benign GCT may show elevated } \\
\text { choline levels on proton MR } \\
\text { spectroscopy [46] }\end{array}$ & $\begin{array}{l}\text { May be related to the degree of their local } \\
\text { aggressiveness }\end{array}$ & Use radiographs and conventional MRI for diagnosis \\
\hline
\end{tabular}

USPIO: ultra-small superparamagnetic iron oxide particles

RES: reticuloendothelial system 
Fig. 28 L4-L5 spondylodiscitis: Sagittal T2 (a) and sagittal CEMRI (b) show intervertebral disk and body endplate destruction (arrows in $\mathbf{a}$ and $\mathbf{b}$ ), oedema of the paraspinal musculature and a large liquid collection inferiorly. The infection extends posteriorly into the spinal canal. Primary bone lesions usually do not contiguously involve adjacent vertebrae
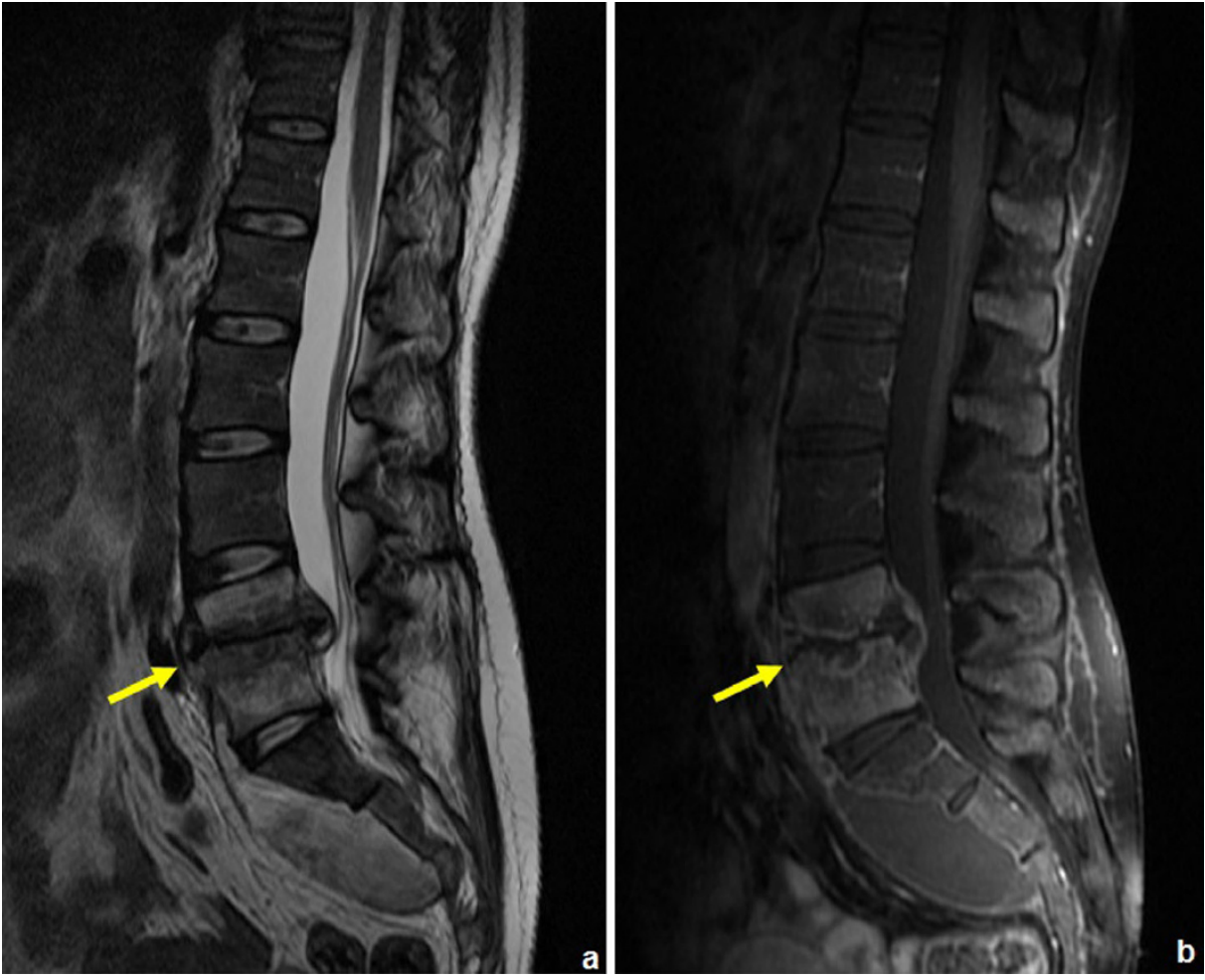

Response to preoperative chemotherapy in bone sarcomas can be classified as good ( $\geq 90 \%$ tumour necrosis) or poor ( $<90 \%$ tumour necrosis) [39]. Decreasing extent of marrow invasion, reduction of tumour volume and a decrease in the amount of associated oedema are favourable conventional MRI indicators (Fig. 25). Quantitative dynamic MRI can estimate the amount of necrosis in bone tumours (viable tumour enhances faster than nonviable tumour and posttreatment changes [40]). If confirmed to be of clinically predictive value, the post-processing software needed for performing this technique would likely become more available [4]. Favourable indicators in dynamic CEMRI are reduction in the slope of the time intensity curve, of the maximum
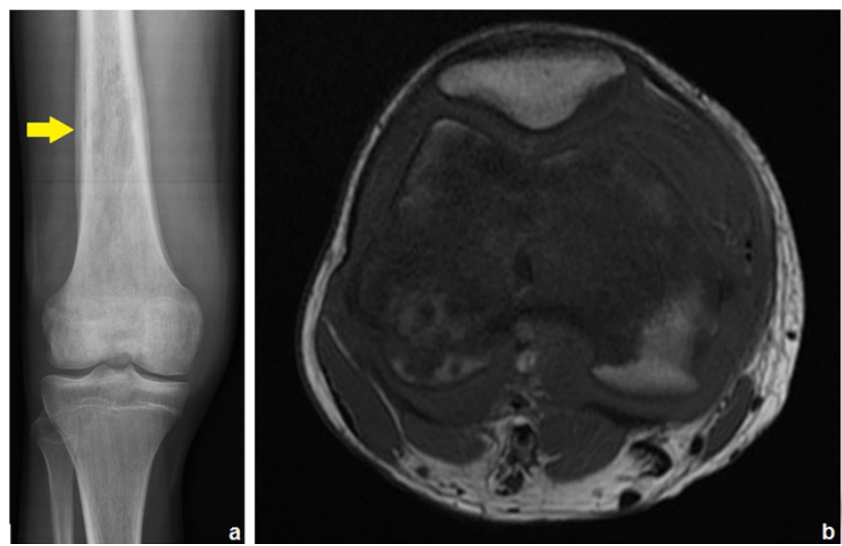

Fig. 29 Acute osteomyelitis radiographic findings (a) can be aggressive and similar to those of Ewing's sarcoma (permeating lesion with periosteal reaction, arrow in a). MRI however shows diffuse soft-tissue enhancement and in the washout rate. These can change the type of signal enhancement time curve [4].

Necrotic tumour does not restrict the movement of water molecules (this translates into low signal intensity in high $b$ values and a high ADC in DWI) as opposed to viable tumour.

\section{MRI applied in follow-up}

Evaluation for osseous metastatic disease should only be done in symptomatic patients [10]. Coronal whole-body and sagittal spine MRI using T1WI and STIR were shown to be

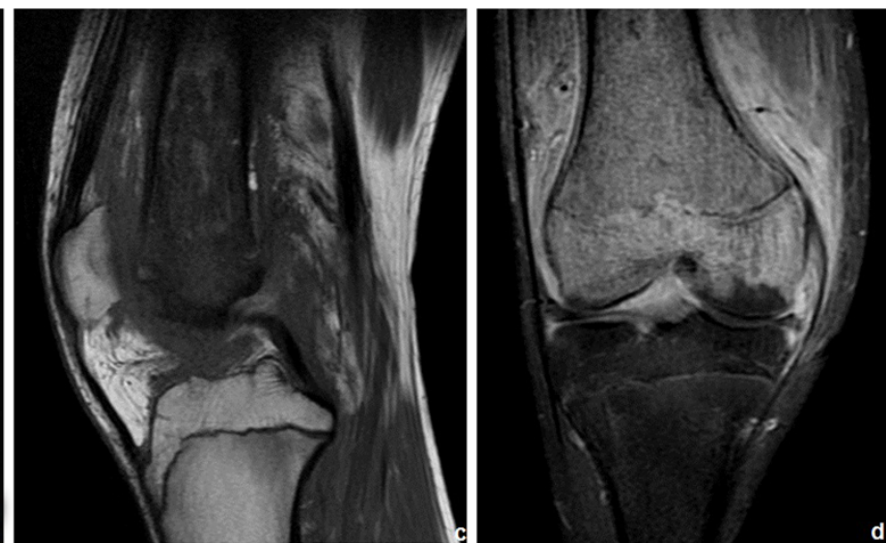

swelling, with no well-defined soft tissue mass (b axial T1WI; c sagittal T1WI; d contrast-enhanced T1WI) 
Fig. 30 Sagittal T1- and T2WI of a typical eosinophilic granuloma of the spine (vertebra plana): preservation of terminal end plates, disks and posterior elements
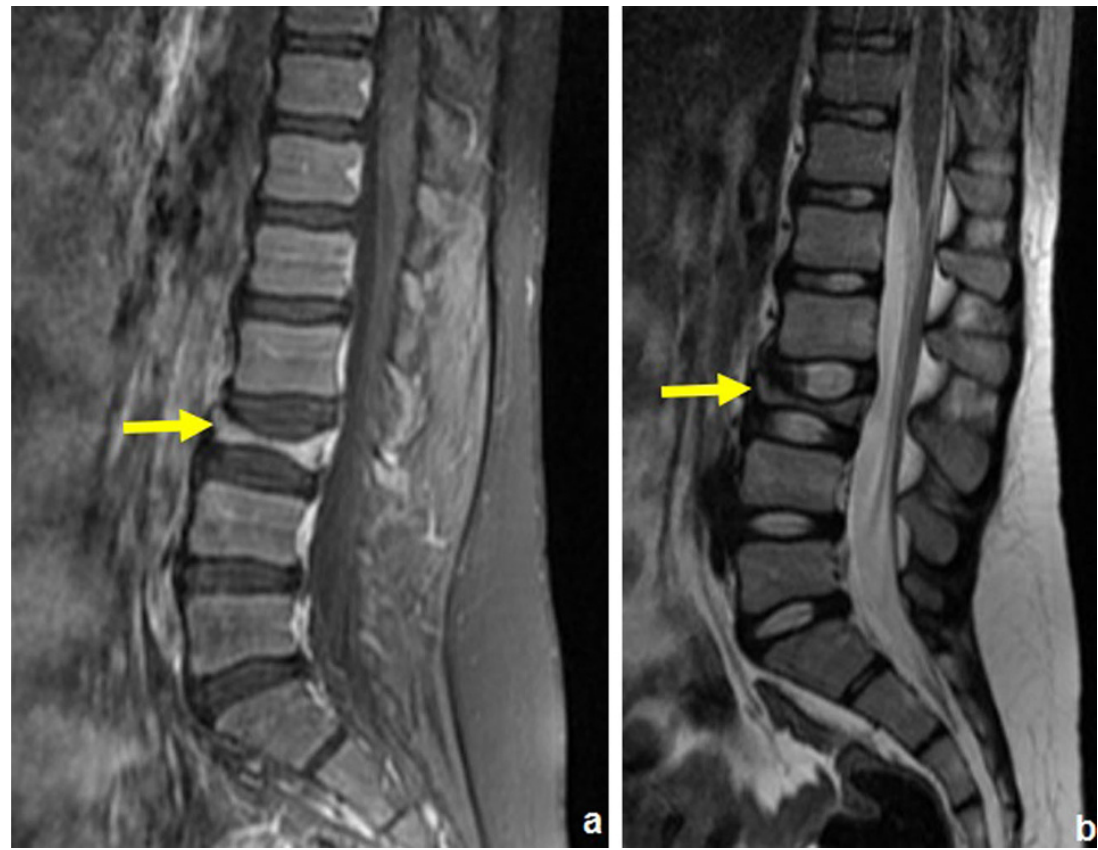

superior to FDG-PET/CT for this purpose [41]. The combined use of radiographs and MRI is strongly recommended for the surveillance for local recurrences [10]. Baseline imaging should be obtained within 3-6 months of definite resection using MRI [19]. Recommended imaging follow-up intervals vary according to whether the tumour is low or high risk [10].

\section{Detection of recurrences and distinction from posttherapeutic changes}

Rooser et al. [42] demonstrated marginal excision, tumour necrosis and extracompartmental extension to be the most important risk factors for local recurrences.

Recurrences should be suspected when residual bone changes occur, such as marrow replacement, cortical

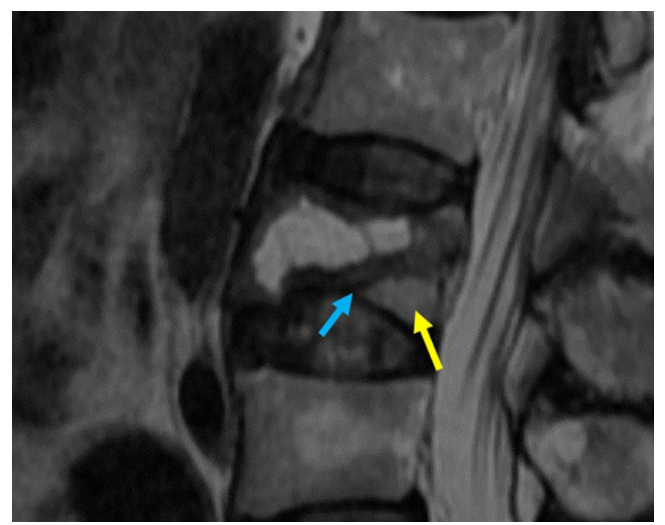

Fig. 31 Sagittal T2WI: typical benign insufficiency vertebral fracture with a visible fracture line (blue arrow), areas of normal marrow (yellow arrow), and no focal lesion or associated mass disruption and osseous destruction. When performing an MRI follow-up study, lesions should first be evaluated according to their T2 signal intensity: if it shows low signal intensity on T2, it generally does not represent recurrent tumour (sensitivity $96 \%$ ) [10]. If it shows high signal on T2 and surgery was the only treatment performed, the likelihood of recurrence is high. When radiotherapy was also carried out, the high signal on T2 is nonspecific for distinguishing recurrence or radiation-induced inflammation [43].

Postoperative fluid collections (haematoma and seroma) can be distinguished from residual tumour or inflammation by means of CEMRI (Figs. 26 and 27): the former will show only thin linear peripheral enhancement.

Chronic, post-therapeutic changes usually lack high signal intensity on T2WI. The presence of vascularised granulation tissue, neovascularity in necrotic areas or reactive hyperaemia can cause gadolinium-based contrast medium enhancement. However, because of its greater vascularisation, tumour tissue normally enhances more.

Dynamic CEMRI may be beneficial by demonstrating early enhancement in tumour tissue that is not seen in posttherapeutic changes or inflammation.

\section{Important diagnostic differentials and mistakes that should be avoided}

There are many nontumoral (e.g. infectious and traumatic) conditions that can look like primary bone tumours ("mimickers"). There is also a group of situations that can lead to errors in staging or incorrect 
Fig. 32 Stress fracture of the calcaneus: sagittal and axial STIR shows an area of localised marrow oedema (arrow in a) and the hypointense fracture line (arrow in b) extending from the cortical to the medullary area
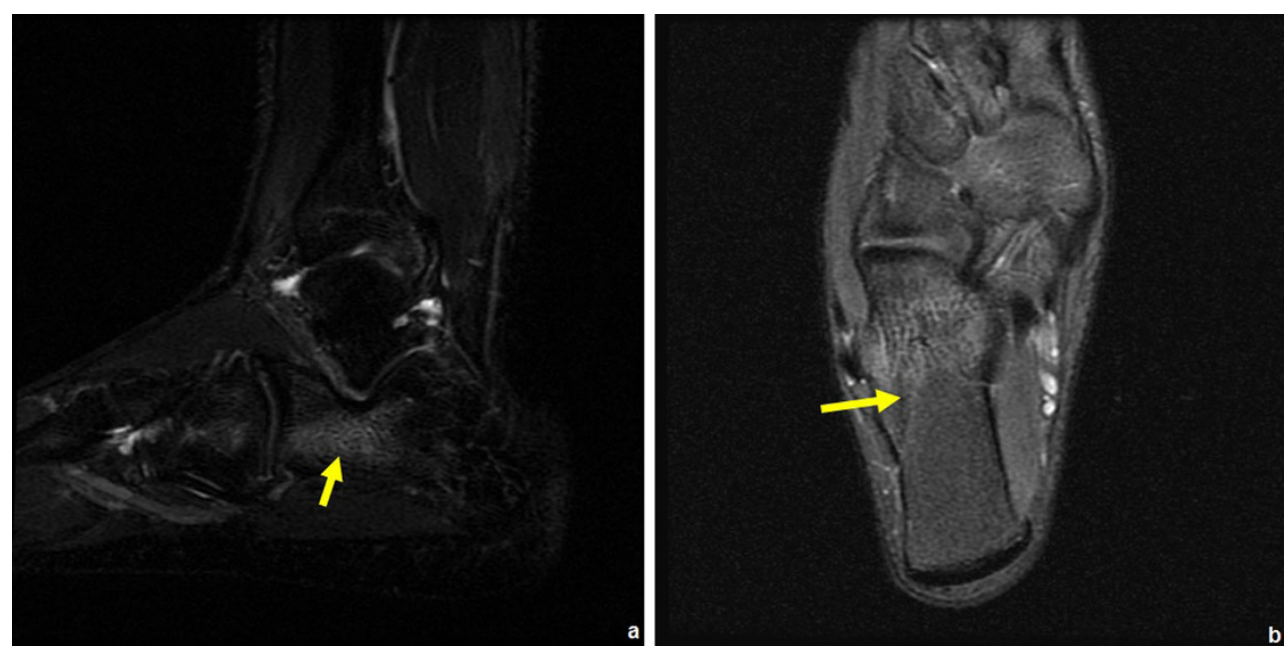

Fig. 33 Acute/intermediate justacortical myositis ossificans of the right thigh: axial STIR (a) shows a localised high signal intensity area with associated oedema. Contrast-enhanced T1WI (b) demonstrates enhancement, which is typical of active lesions. This lesion does not disrupt the cortical area
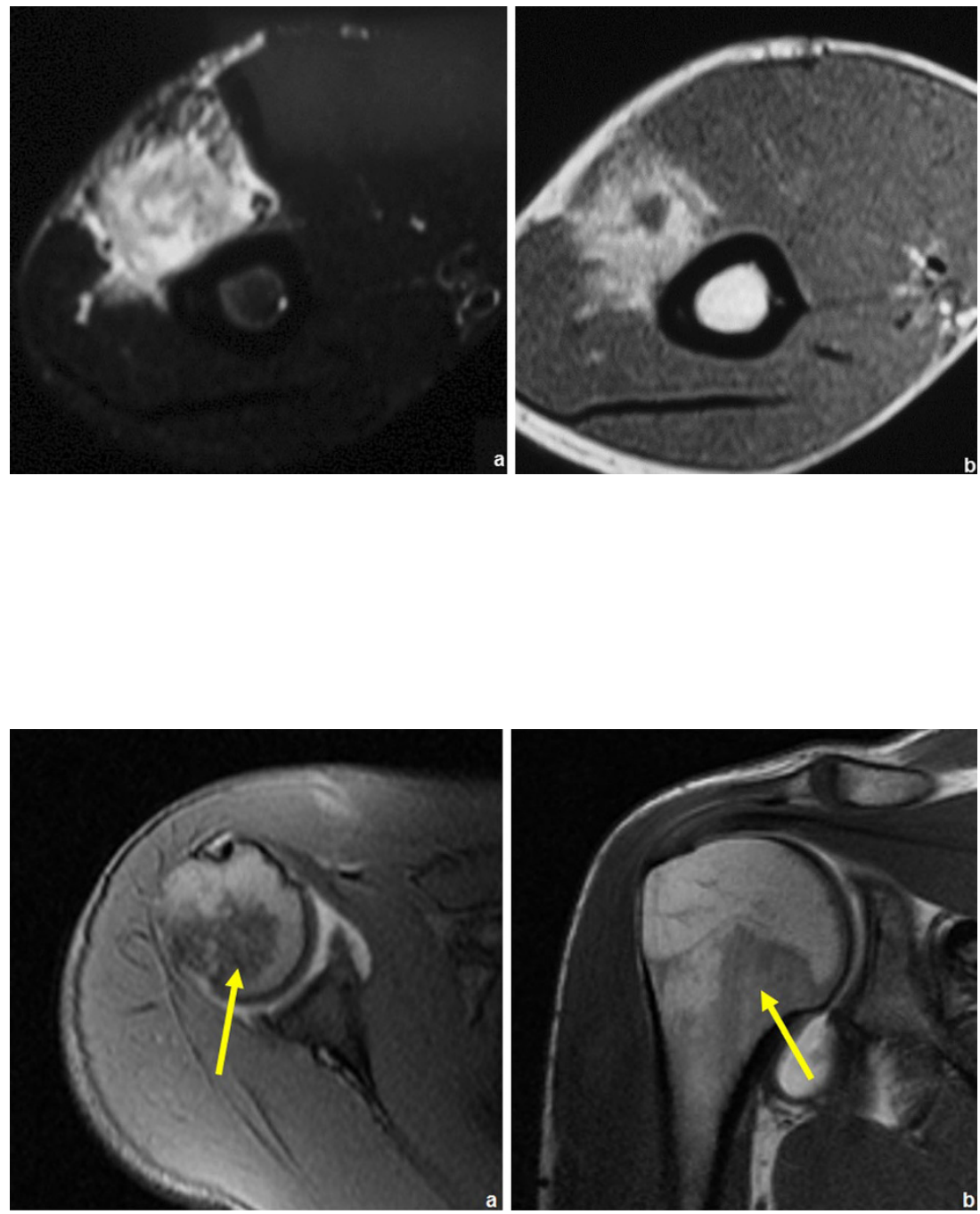

Fig. 34 Normal haematopoietic marrow in the proximal humerus (arrows in $\mathbf{a}$ and b). In children, the normal bone marrow is highly cellular (low signal on T1WI and high signal T2 FS WI). With increasing age, a gradual conversion from this highly cellular marrow to fatty marrow occurs (with an increase in the bone marrow signal on T1WI and a decline on T2 FS WI). In long bones, this conversion first involves the epiphyses, then the diaphyses and finally the metaphyses [24] 
Fig. 35 A patient with a history of lung cancer: coronal STIR (a) showed several suspicious areas on both tibial bones (arrows in a), which could be misinterpreted as distant recurrence if not noticed to show higher T1 (arrows in $\mathbf{b}$ and c) signal than adjacent muscle. Posterior follow-up (d) showed complete resolution of these areas. This reconversion occurs in a reverse fashion compared to the conversion from haematopoietic marrow to fatty marrow (i.e. the reconversion progresses from the central skeleton to the periphery) [24]
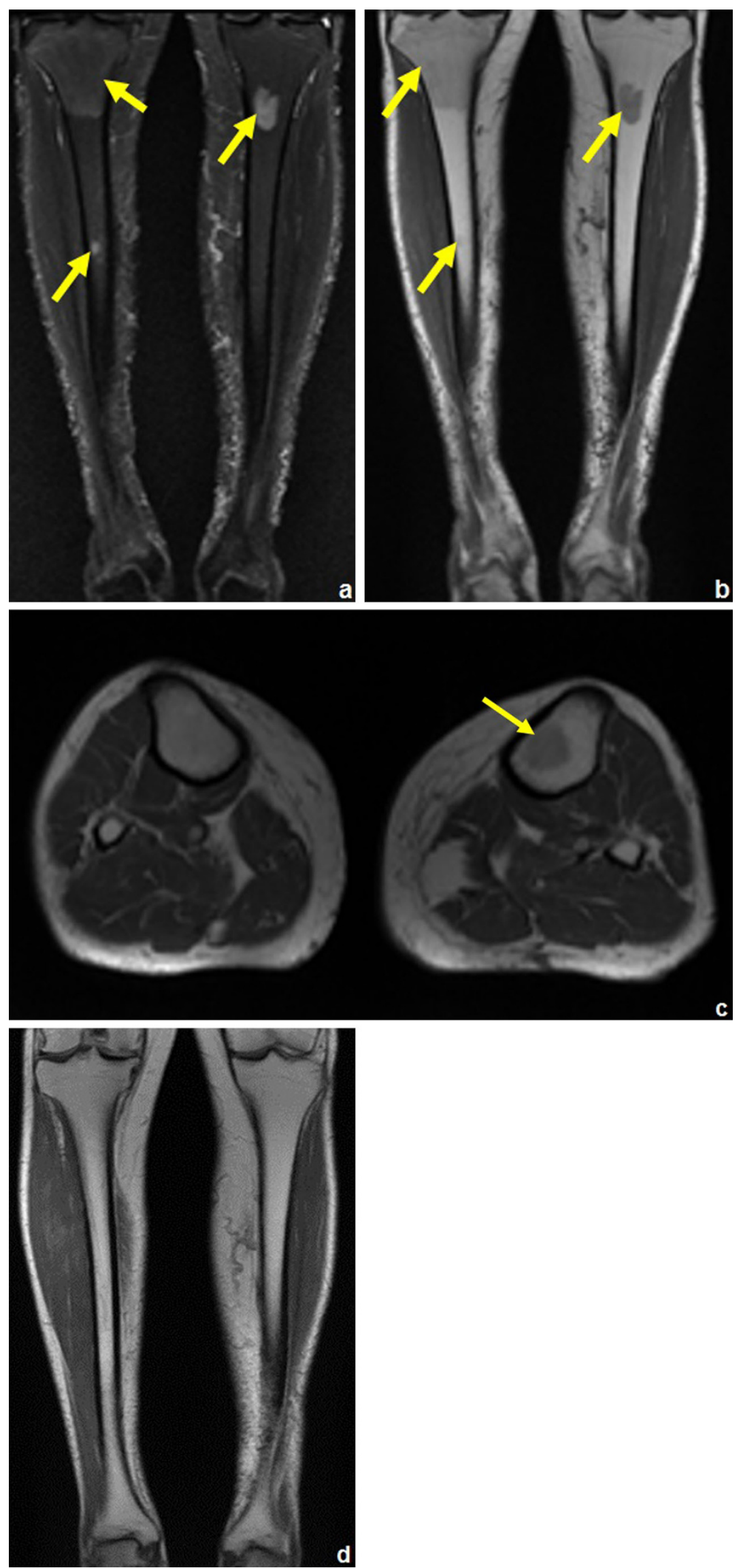


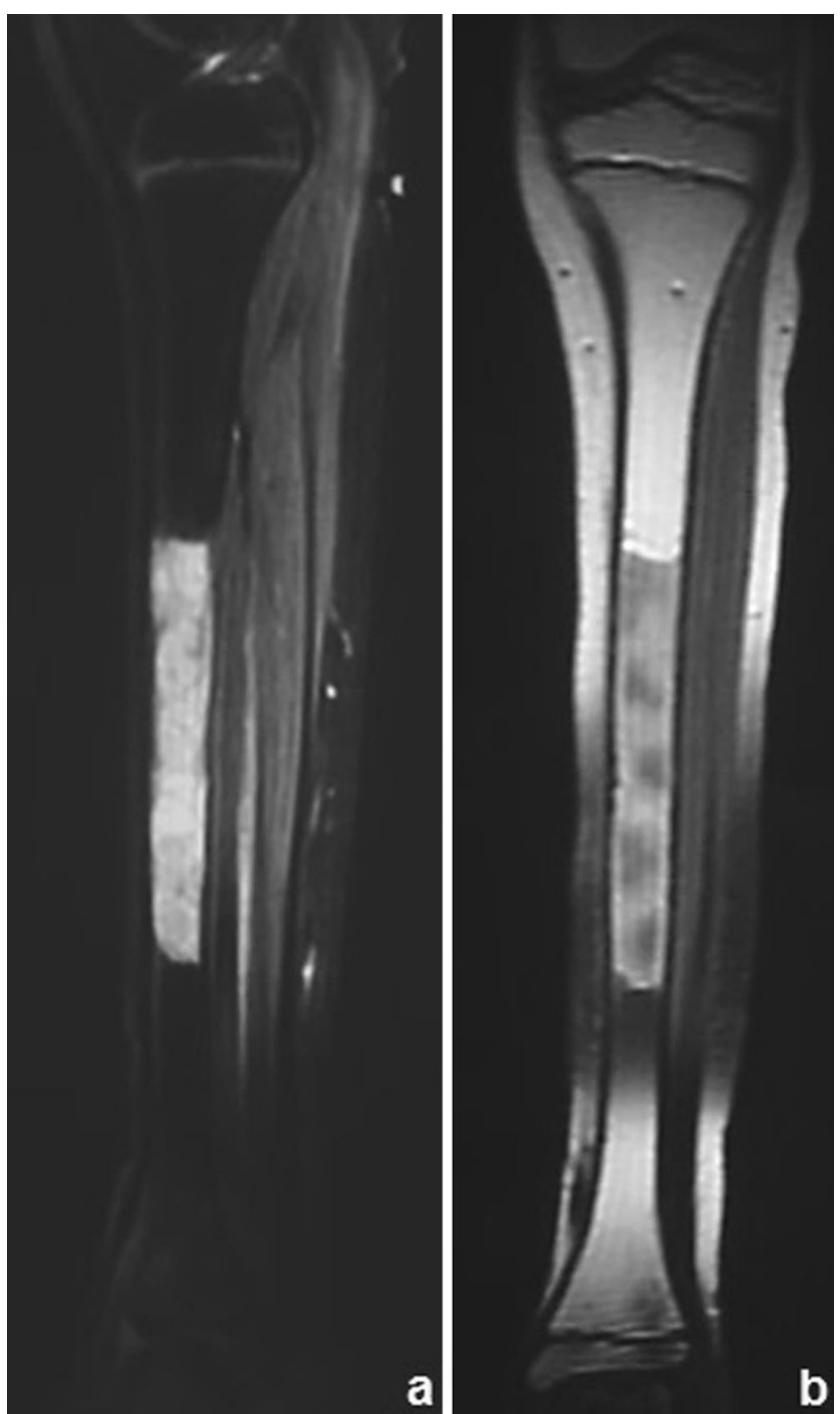

Fig. 36 Medullary rebound in a young female patient with a history of recent chemotherapy for treatment of Ewing's sarcoma: coronal STIR (a) showed an area of increased intensity in the tibial diaphysis that displayed higher signal intensity than the muscle on T1WI (b)

diagnosis of recurrence (and thus can be regarded as pitfalls). Although their detailed discussion is beyond the scope of this work, some of them should be mentioned and are briefly discussed in Tables 7 and 8 and (Figs. 28, 29, 30, 31, 32, 33, 34, 35, and 36) [15, 19, 24, 44-46].

\section{Limitations}

General MRI contraindications also apply to the evaluation of bone tumours and tumour-like conditions (e.g. patient size, clinical status, cardiac pacemaker).

Low-risk Gd-based contrast agents [47] should only be used if they can provide essential diagnostic information in patients with a glomerular filtration rate $<30 \mathrm{ml} / \mathrm{min}$ and in pregnant women [47].

Tumour recurrence may be hard to detect when orthopaedic implants are in close proximity to tumour sites because of susceptibility artefacts.

MRI is limited in evaluating calcifications. CT plays a further role in the characterisation of sclerotic or mixed (lytic/sclerotic) lesions and is superior to MRI in the evaluation of osteoid osteoma [10, 35].

\section{Conclusion}

A good knowledge of the characteristic MRI findings of benign and malignant osseous conditions and their role in staging, therapeutic planning and follow-up in the setting of malignancy is essential for optimal patient care.

Open Access This article is distributed under the terms of the Creative Commons Attribution License which permits any use, distribution, and reproduction in any medium, provided the original author(s) and the source are credited.

\section{References}

1. American College of Radiology (2013) ACR Appropriateness Criteria. Primary Bone Tumors. American College of Radiology. Available via http://www.acr.org/ /media/ACR/Documents/ AppCriteria/Diagnostic/PrimaryBoneTumors.pdf. Accessed 9 Apr 2014

2. Ojala R, Sequeiros RB, Klemola R, Vahala E, Jyrkinen L, Tervonen O (2002) MR-guided bone biopsy: preliminary report of a new guiding method. J Magn Reson Imaging 15(1):82-86

3. Ribeiro MB, Oliveira CR, Filippi RZ et al (2009) Estudo histopatológico do trajeto de biópsia de tumores musculoesqueléticos malignos. Acta Ortopédica Brasileira 17(5):279-281

4. Davies AM, Sundaram M, James SLJ (2009) Imaging of Bone Tumors and Tumor-Like Lesions (Techniques and Applications). Springer, Berlin Heidelberg

5. Erly WK, Oh ES, Outwater EK (2006) The utility of in-phase/ opposed-phase imaging in differentiating malignancy from acute benign compression fractures of the spine. AJNR Am J Neuroradiol 27(6):1183-1188

6. Disler DG, McCauley TR, Ratner LM, Kesack CD, Cooper JA (1997) In-phase and out-of-phase MR imaging of bone marrow: prediction of neoplasia based on the detection of coexistent fat and water. AJR Am J Roentgenol 169(5):1439-1447

7. Berquist TH (1993) Magnetic resonance imaging of primary skeletal neoplasms. Radiol Clin North Am 31(2):411-24

8. Hayashida Y, Yakushiji T, Awai K et al (2006) Monitoring therapeutic response of primary bone tumors by diffusion weighted image: initial results. Eur Radiol 16(12):2637-2643

9. Bley TA, Wieben O, Uhl M (2009) Diffusion-weighted MR imaging in musculoskeletal radiology: applications in trauma, tumors, and inflammation. Magn Reson Imaging Clin N Am 17(2):263-275

10. American College of Radiology (2011) ACR Appropriateness Criteria. Follow-up of Malignant or Aggressive Musculoskeletal Tumors. Available via http://www.acr.org/ / 
media/ACR/Documents/AppCriteria/Diagnostic/ FollowupMalignantOrAggressiveMusculoskeletalTumors.pdf. Accessed 9 Apr 2014

11. Wang CK, Li CW, Hsieh TJ, Chien SH, Liu GC, Tsai KB (2004) Characterization of bone and soft-tissue tumors with in vivo $1 \mathrm{H}$ MR spectroscopy: initial results. Radiology 232(2):599-605

12. May DA, Good RB, Smith DK, Parsons TW (1997) MR imaging of musculoskeletal tumors and tumor mimickers with intravenous gadolinium: experience with 242 patients. Skeletal Radiol 26(1):2-15

13. Zimmer WD, Berquist TH, McLeod RA et al (1985) Bone tumors: magnetic resonance imaging versus computed tomography. Radiology 155(3):709-718

14. Simpfendorfer CS, Ilaslan H, Davies AM, James SL, Obuchowski NA, Sundaram M (2008) Does the presence of focal normal marrow fat signal within a tumor on MRI exclude malignancy? An analysis of 184 histologically proven tumors of the pelvic and appendicular skeleton. Skeletal Radiol 37(9):797-804

15. Morrison WB, Sanders TG (2008) Problem Solving Musculoskeletal Imaging, 1st edn. Mosby Elsevier, Philadelphia, pp 251-324

16. Greenspan A (2011) Orthopedic imaging: a practical approach, 5th edn. Lippincott Williams \& Wilkins

17. Alyas F, James SL, Davies AM, Saifuddin A (2007) The role of MR imaging in the diagnostic characterization of appendicular bone tumours and tumour-like conditions. Eur Radiol 17:2675-86

18. Woertler K, Lindner N, Gosheger G, Brinkschmidt C, Heindel W (2000) Osteochondroma: MR imaging of tumor-related complications. Eur Radiol 10(5):832-840

19. Manaster BJ, Petersilge CA, Roberts CC, Hanrahan CJ, Moore S (2010) Diagnostic Imaging: Musculoskeletal-Non-Traumatic Disease, 1st edn. Amirsys Publishing, Philadelphia, pp 2-1 - 2-229

20. Alyas F, Saifuddin A (2008) Fluid-fluid levels in bone neoplasms: variation of $\mathrm{T} 1$-weighted signal intensity of the superior to inferior layers - diagnostic significance on magnetic resonance imaging. Eur Radiol 18:2642-2651

21. Van Dyck P, Vanhoenacker FM, Vogel J et al (2006) Prevalence, extension and characteristics of fluid-fluid levels in bone and soft tissue tumors. Eur Radiol 16(12):2644-51

22. Murphey MD, Wan Jaovisidha S, Temple HT, Gannon FH, Jelinek JS, Malawer MM (2003) Telangiectatic osteosarcoma: radiologicpathologic comparison. Radiology 229(2):545-553

23. Jee WH, Park YK, McCauley TR et al (1999) Chondroblastoma: MR characteristics with pathologic correlation. J Comput Assist Tomogr 23(5):721-726

24. Daldrup-Link HE, Henning T, Link TM (2007) MR imaging of therapy-induced changes of bone marrow. Eur Radiol 17(3):743-761

25. Tamburrini O, Cova MA, Console D, Martingano P (2007) The evolving role of MRI in oncohaematological disorders. Radiol Med 112(5):703-21

26. Narquin S, Ingrand P, Azais I et al (2013) Comparison of whole-body diffusion MRI and conventional radiological assessment in the staging of myeloma. Diagn Interv Imaging 94(6):629-36. doi:10.1016/j. diii.2013.01.005

27. Schirrmeister $H$, Bommer $M$, Buck $A K$ et al (2002) Initial results in the assessment of multiple myeloma using 18 F-FDG PET. Eur J Nucl Med Mol Imaging 29(3):361-6

28. Lecouvet FE, Malghem J, Michaux L et al (1999) Skeletal survey In advanced multiple myeloma: radiographic versus MR imaging survey. Br J Haematol 106:35-39

29. Durie BG, Kyle RA, Belch A et al (2003) Myeloma management guidelines: a consensus report from the Scientific Advisors of the International Myeloma Foundation. Hematol J 4(6):379-398
30. Walker R, Barlogie B, Haessler J et al (2007) Magnetic resonance imaging in multiple myeloma: diagnostic and clinical implications. $\mathrm{J}$ Clin Oncol 25:1121-1128

31. Dimopoulos M, Terpos E, Comenzo RL et al (2009) International myeloma working group consensus statement and guidelines regarding the current role of imaging techniques in the diagnosis and monitoring of multiple myeloma. Leukemia 23(9):1545-1556

32. Rajkumar SV (2005) MGUS and smoldering multiple myeloma: update on pathogenesis, natural history, and management. Hematology Am Soc Hematol Educ Program 1:340-3455

33. Lecouvet FE, Vande Berg BC, Michaux L et al (1998) Stage III multiple myeloma: clinical and prognostic value of spinal bone marrow MR imaging. Radiology 209(3):653-60

34. Dutoit JC, Vanderkerken MA, Verstraete KL (2013) Value of whole body MRI and dynamic contrast enhanced MRI in the diagnosis, follow-up and evaluation of disease activity and extent in multiple myeloma. Eur J Radiol 82(9):1444-52. doi:10.1016/j.ejrad.2013.04.012

35. Hogeboom WR, Hoekstra HJ, Mooyaart EL et al (1992) MRI or CT in the preoperative diagnosis of bone tumours. Eur J Surg Oncol 18(1):67-72

36. Sajadi KR, Heck RK, Neel MD et al (2004) The incidence and prognosis of osteosarcoma skip metastases. Clin Orthop Relat Res 426:92-96

37. American Joint Committee on Cancer (2010) AJCC Cancer Staging Manual, 7th edn. Springer, New York Dordrecht Heidelberg London, pp 279-290

38. Munshi NC, Longo DL, Anderson KC (2011) Plasma Cell Disorders. In: Longo D.L., Kasper D.L., Jameson J.L., Fauci A.S., Hauser S.L., Loscalzo J.(eds) Harrison's Principles of Internal Medicine, 18th edn. McGraw-Hill Professional, pp 936-944

39. Bacci G, Bertoni F, Longhi A et al (2003) Neoadjuvant chemotherapy for high-grade central osteosarcoma of the extremity. Histologic response to preoperative chemotherapy correlates with histologic subtype of the tumor. Cancer 97(12):3068-3075

40. Amit P, Patro DK, Basu D, Elangovan S, Parathasarathy V (2013) Role of dynamic MRI and clinical assessment in predicting histologic response to neoadjuvant chemotherapy in bone sarcomas. Am J Clin Oncol. doi:10.1097/COC. 0b013e31827b4f6f

41. Schmidt GP, Schoenberg SO, Schmid R (2007) Screening for bone metastases: whole-body MRI using a 32-channel system versus dualmodality PET-CT. Eur Radiol 17(4):939-949

42. Rööser B, Attewell R, Berg NO, Rydholm A (1987) Survival in soft tissue sarcoma. Prognostic variables identified by multivariate analysis. Acta Orthop Scand 58(5):516-522

43. Vanel D, Lacombe MJ, Couanet D, Kalifa C, Spielmann M, Genin J (1987) Musculoskeletal tumors: follow-up with MR imaging after treatment with surgery and radiation therapy. Radiology 164(1):243245

44. Kransdorf MJ, Murphey MD (2014) Imaging of Soft Tissue Tumors, 3rd edn. Lippincott williams \& wilkins, pp 542-549

45. Grey AC, Davies AM, Mangham DC, Grimer RJ, Ritchie DA (1998) The 'penumbra sign' on T1-weighted MR imaging in subacute osteomyelitis: frequency, cause and significance. Clin Radiol 53(8): 587-92

46. Sah PL, Sharma R, Kandpal H et al (2008) In vivo proton spectroscopy of giant cell tumor of the bone. AJR Am J Roentgenol 190(2): W133-139

47. European Society of Urogenital Radiology (2013) ESUR Guidelines on Constrast Media. Available via http://www.esur. org/guidelines/. Accessed 9 Apr 2014 